\title{
Prediction of mechanical properties of knitted fabrics under tensile and shear loading: mesoscale analysis using representative unit cells and its validation
}

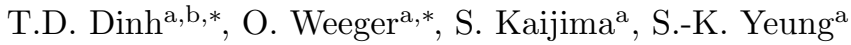 \\ ${ }^{a}$ Singapore University of Technology and Design, SUTD Digital Manufacturing and Design Center, 8 Somapah Road, Singapore 487372, \\ Singapore \\ ${ }^{b}$ Department of Materials, Textiles and Chemical Engineering (MaTCh), Faculty of Engineering and Architecture, Ghent University, Tech \\ Lane Ghent Science Park-Campus A, Technologiepark-Zwijnaarde 903, B-9052, Belgium
}

\begin{abstract}
This article presents a numerical framework to predict the mechanical behavior of knitted fabrics from their discrete structure at the fabric yarn level, i.e., the mesostructure, utilizing the hierarchical multiscale method. Due to the regular distribution of yarn loops in a knitted structure, the homogenization theory for periodic materials can be employed. Thus, instead of considering the whole fabric sample under loading, a significantly less computationally demanding analysis can be done on a repeated unit cell (RUC). This RUC is created based on simple structural parameters of knitted yarn loops and its fabric yarns are assumed to behave transversely isotropic. Nonlinear finite element analyses are performed to determine the stress fields in the RUC under tensile and shear loading. During this analysis, contact friction among yarns is considered as well as the periodic boundary conditions are employed. The macroscopic stresses then can be derived from the stress fields in the RUC by means of the numerical homogenization scheme. The physical fidelity of the proposed framework is shown by the good agreement between the predicted mechanical properties of knitted fabrics and corresponding experimental data.
\end{abstract}

Keywords: knitted fabrics, multiscale analysis, numerical homogenization method, periodic boundary conditions.

\section{Introduction}

There has been increasing interest in the mechanical ${ }_{25}$ behavior of fabric materials, due to the wide range of applications they can be utilized in. Especially textile com5 posite materials, where fabric structures are utilized as preforms, are gaining more and more attention, beyond the traditional textile industries, i.e., apparel and fashion. Indeed, textile composite materials can now be found in domains such as aerospace, automotive and submarines 10 industries, as well as architecture and civil engineering, e.g., tension membrane structures [1]. Furthermore, socalled functional, technical or smart textiles are utilized ${ }_{35}$ in biomedical applications, defense and flexible electronics [2].

In general, woven, non-woven, braided and knitted fabrics can be distinguished according to their method of fabrication. Among them, knitted fabrics, which are pro- ${ }_{40}$ duced by intermeshing loops of yarns using knitting needles, have so far the most modest percentage for usage in technical applications [3, even though they are widely utilized for outerwear, such as dresses and sportswear due to their excellent formability [4].

\footnotetext{
* Corresponding authors

Email addresses: Tiendung. Dinh@uGent. be (T.D. Dinh), Oliver_Weeger@sutd.edu.sg (O. Weeger)
}

However, due to the advancement in knitting technology as well as the availability of high performance fibers such as carbon, glass and aramid, knitted fabrics are gaining more and more interest in different innovative applications. For example, smart fibers are used as the base material for active knitted actuators [5] or shape memory alloy (SMA) fibers are knitted into garments to create the shape changing cloths [6. In addition, knitted textiles are also the materials of choice in biomaterials because of their high flexibility and low tendency to fray 7 . Moreover, in the composite materials industry, the utilization of knitted fabrics as preforms was considered skeptically because of their relatively low stiffness and strength originating from their low fiber volume fraction. Nevertheless, the curved nature of the knitted loops manifests itself in the outstanding drapability of the resultant knitted fabrics, which catches the attention of composite materials engineers. This special property enables knitted fabric to be utilized in forming complex and deeply curved composite components 8 .

Additionally, changing fiber/yarn materials and knitting pattern of preforms will vary the mechanical prop45 erties of the knitted fabric composites. Therefore, with the currently available fiber materials and various knitting patterns, which can be formed from modern knitting machines, composite engineers indeed have more options to tailor the mechanical properties of knitted composites 
50 in such a way that they can fit a particular application.105 Especially, knitted fabrics can be utilized to create 3D preforms with several types of fiber and various local knitting patterns. As a consequence, various local thicknesses and properties preforms can be achieved [8. This advantage

${ }_{55}$ has for instance been exploited to create auxetic fabrics ${ }_{110}$ and auxetic composites, which have negative Poisson's ratio 9 .

From production point of view, compared to other kinds of fabrics, knitted fabrics do have several advantages such

60 as mass productivity, low fabrication cost and quick set-up ${ }_{115}$ of the knitting machines 3,8 . It is noteworthy that the stiffness and strength of knitted fabrics can be improved by introducing straight yarns as described in [10].

It was experimentally validated that the mechanical

65 properties of dry fabric preforms have a strong effect on 120 the mechanical properties of the resultant textile composites [8, 11. In order to fully exploit the advantages as well as to identify the limitation of knitted fabrics, a better understanding of the mechanical properties of this kind of 70 materials is needed, not only in composite industry but ${ }_{125}$ also in other industries, such as computer graphics 4], apparel and industrial applications 12. Fibrous materials in general and knitted fabrics in particular exhibit high level of anisotropy and nonlinearity in mechanical properties due to the heterogeneous nature of their meso/micro structures [13. The macroscopic mechanical behavior of ${ }_{130}$ knitted fabrics is mainly governed by the properties of the constituent fibers and the intricate interactions among fibers, i.e., intra-yarn contact as well as among yarns, i.e., inter-yarn contract 14. Thus, modeling and simulation of knitted fabrics is a very challenging task.

The outline of this manuscript is as follows: in the next section, a detailed literature review of knitted fabrics modeling is conducted, including both geometrical and 85 mechanical modeling aspects. Afterward, the ingredients of our proposed model, viz., the geometrical model of the ${ }_{140}$ plain knitted unit cell, as well as the mechanical material properties of the fabric yarn are presented. Subsequently, the elements of the Repeated Unit Cell (RUC)-

90 based homogenization technique and their implementation in ABAQUS/Standard are mentioned. This section is fol-145 lowed by numerical results, including the successful validation of our computational approach against experimental references. The manuscript ends with some concluding 95 remarks.

\section{Literature study on knitted fabrics modeling}

During last few decades, the research in textile composites and dry fabric materials has been very active. However, most of the efforts have been focusing on woven fab-155 rics, while significantly less references exist on the investigation of material behavior of knitted fabrics. As a whole, mechanical properties of knitted fabrics are significantly different from those of woven fabrics. The main reason is that woven fabrics are made by interlacing slightly crimped ${ }_{160}$ fibers orthogonally. As a consequence, they possess very little stretchability [15. On the other hand, knitted fabrics are produced by looping the yarn through itself to make a chain of stitches that are subsequently connected together. The curvature of the yarn in the knitting pattern is the source of stretchability of knitted fabrics, even in case they are made of high modulus fibers [16. In fact, knitted fabrics possess a hierarchical multiscale nature. The fabric is composed of the yarn and the yarn is composed of the fibers. Therefore, modeling of knitted fabrics can be addressed at three different scales, viz., macroscale or fabric scale, mesoscale or yarn scale, and microscale or fiber scale. Hitherto, most of the works in modeling knitted fabrics have been proposed at mesoscale [10, 17- 19]. To model knitted fabrics at mesoscale level, we need to define a realistic geometry model of the repeated unit cell (RUC), which is the smallest pattern used to represent the whole fabric by several translations, a material constitutive law for the fabric yarn, which can reflect its fibrous nature [17, as well as frictional contact among the yarns, and appropriate boundary conditions.

\subsection{Geometrical models}

In the literature, a couple of geometrical models were proposed for knitted fabrics, mainly aiming at plain weft knitted fabrics. In a plain weft knitted structure, rows are referred to as courses, which run across the width of the fabric, and columns are referred to as wales, which run along the length of fabric ( $c f$. fig. 1). According to Gravas et al. 20, the first two-dimensional geometrical model for knitted fabrics was proposed by Tompkins [21 more than a hundred years ago. A few years later, Chamberlain [22] proposed another two-dimensional model. That model presented a projection of the yarn loop of a plain weft knitted fabric, which is composed of circular arcs and straight lines. The first three-dimensional model for plain knitted fabric was proposed by Peirce [23. In that model, Peirce assumed that the heart line of the course yarn is composed of circular arcs and straight lines. Additionally, he assumed that this heart line lies on a circular cylindrical surface. It was pointed out in the paper of Leaf and Glaskin 24 that the geometrical model of the fabric yarn of Peirce caused discontinuities in torsion that occur at material points in the loop. Thus, they proposed another model [24] to fix that defect. In that proposed model, Leaf and Glaskin assumed that the projection of the heart line of the yarn is composed of only circular arcs. That model was later utilized in 25] to model knitted fabric reinforced composites.

Recently, Vassiliadis [26] proposed a more complex geometrical model for plain weft knitted fabrics using BSplines. The inputs of this model are the main structural parameters of a single jersey fabric, viz., the course space, the wale space and the thickness of the yarn. This geometrical model was utilized in mesostructural analyses by the same author 18. Moreover, the model was also employed in the analyses presented in [27, 28]. Furthermore, based 
on that model Abghary et al. 10 have proposed a novel 200 geometrical model for $1 \times 1$ rib knitted fabrics. Recently, McKee et al. 15] have utilized trigonometric functions to describe the heart line of the knitted yarn. The parameters in that model were calibrated with X-ray tomographic images. It is noteworthy that all of the geometrical models 205 mentioned thus far were aimed for relaxed knitted fabrics. Therefore, the residual stresses originated from the knitting process mentioned in [29] can be neglected.

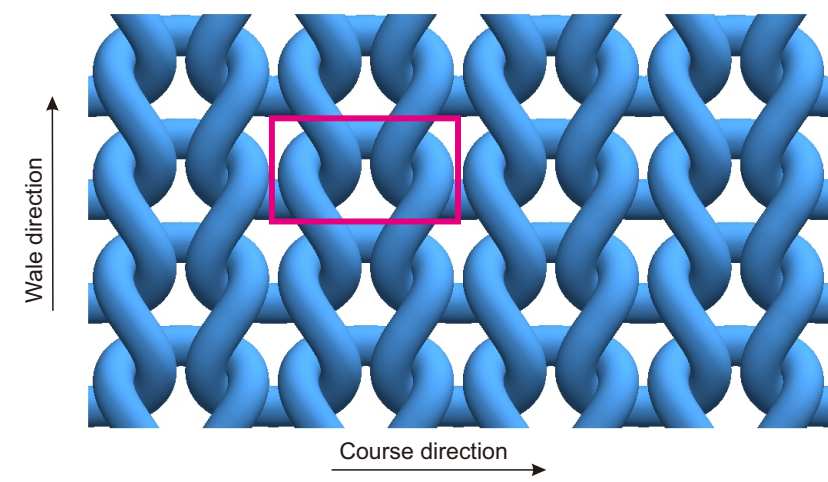

210

Figure 1: Plain weft-knitted structure and its RUC bounded by the rectangle.

\subsection{Mechanical models}

There have been various approaches to simulate the mechanical behavior of knitted fabrics. As mentioned in [29], during the course of loading the main deformation modes of knitted yarns are inter-yarn slip, inter-yarn shear,230 yarn bending, yarn buckling, intra-yarn slip, yarn stretching, yarn compression and yarn twist. These deformation mechanisms can indeed be captured at the yarn level, i.e., the mesoscale. Therefore, it is no surprise that most of the models for knitted fabrics were proposed at that length ${ }_{235}$

There were some attempts to use the Euler-Bernoulli beam model to investigate tensile mechanical properties of knitted yarns analytically, among them are [19, 3033. The most successful model in this approach is the ${ }_{240}$ one proposed by Hong et al. 19 for plain weft knitted fabrics made of high performance fibers, such as glass, carbon and aramid. In their model, they considered a two-dimensional loop structure, which represents the relaxed state of the knitted fabric. The yarn to yarn contact $_{245}$ was replaced by kinematic boundary conditions at contact points. Then the static equilibrium conditions of the fabric under uniaxial tests were established by using the EulerBernoulli beam theory. The theoretical predictions are in good correlation with the experimental data. That model ${ }_{250}$ was later utilized by Abel et al. 34 to predict the actuation response of a shape memory alloy garter knitted fabric and Dusserre et al. 32 to predict the elastic properties of knitted composite reinforcement with inlaid yarns. However, there are several strong assumptions in the model of ${ }_{255}$
Hong et al. 19. Firstly, the change of the yarn's cross section is neglected. This assumption can be accepted in case of fabrics made of monofilament yarns, but it can lead to erroneous results for multifilament fabrics [2]. Additionally, the contact friction between yarns is not considered in that model. As a consequence, that model cannot be used to study the hysteresis effect of knitted fabrics. In order to investigate this effect, Dusserre et al. 33 improved the original model of Hong et al. 19 by introducing the contact friction. However, visible discrepancies between theoretical predictions and the experimental data can be seen from their results. Moreover, the expansion of these analytical models becomes extremely difficult for knitted fabrics that have complicated knitting patterns because of the complexity of mathematical equations needed to describe the fabric behavior.

Ji et al. 35. proposed a mass-spring system to model plain knitted fabrics. In their model, masses are connected to each other by massless springs. These springs are used to resist the in-plane elongation or compression, shearing, and bending deformations. The mechanical properties of these springs were derived from the corresponding experimental tests. This approach is undeniable the method of choice to visualize fabric and garment due to its attractive computational time. However, validation of this model is missing in ref. [35. Another effort from computer graphics community is the work of Wang et al. 4], who employed a data-driven technique to model knitted fabrics with different kinds of knitting patterns. In their model, they used piecewise linear elasticity model to approximate the nonlinear, anisotropic behavior of knitted fabrics. There are 39 parameters in their model and they had to perform 105 tests for each cloth material, which make this model become clumsy for practical utilization. Nevertheless, the obtained results presented in that paper are very encouraging. The fabric's deformation looks quite realistic since especially wrinkling patterns are captured quite well.

Yeoman et al. 7] proposed a phenomenological model for knitted fabrics at macroscale, i.e., fabric level, utilized in biomedical applications. Their model is indeed an expansion of the model proposed by Chuong and Fung 36 for soft biological tissues by including shear and increasing the number and order of coefficients in the strain energy function. That model yields quite good results when compared with the experimental data from uniaxial tensile tests, but its performance is deteriorated when compared with the experimental data from a simple shear test. Those authors also claimed that the performance of their proposed model can be improved if more terms are added into the strain energy function. However, to do so, it demands more experimental data to calibrate the model parameters.

Recently, Fillep et al. [14 employed numerical homogenization method to predict the mechanical behavior of dry plain knitted fabrics. To the best knowledge of the authors, that is the first work in which the numerical homogenization method was utilized for knitted fabrics. 
However, in that paper the validation of the numerical results is missing. Indeed, the numerical homogenization method is very versatile and has been widely utilized to ${ }^{295}$ predict the mechanical properties of woven fabrics [37, woven coated fabrics [13] and textile composites [38 40]. However, thus far there have been very few works in which the numerical homogenization method was used for simulating the mechanical behaviors of knitted fabrics. By employing this method, we can reduce the number of physical experiments, which can be very extensive and expensive. Moreover, it can help to gain a better understanding of the deformation mechanisms of knitted fabrics during the course of loading as well as to investigate the effects of different parameters to the overall macroscopic behavior of knitted fabrics 41. Because of these advantages, numerical homogenization is the method of choice in the present article.

\section{Modeling inputs}

\subsection{Geometrical parameters}

Since the experimental data utilized to validate the numerical simulations in this work are extracted from [18, we also employed the geometrical model proposed in 26 by the same authors for the sake of consistency. Figure 2 presents the idealized 3D unit cell of a plain knitted fabric proposed in [26]. The inputs of this model are the course distance $c$, the wale distance $w$ and the yarn diameter $D$. Other parameters are derived directly from these three parameters, except for the parameter $t$. This parameter is determined in such a way that the length of the yarn loop is minimal. Because of the symmetry of the model, the whole unit cell can be derived once a quarter of the yarn loop, i.e., EMKA in the front view in fig. 2 is determined. This path can be divided into three parts and their math-

- Part EM $(0<y<c / 2)$ :

$$
\begin{aligned}
& x(y)=-\frac{D}{c} y, \\
& z(y)=\sqrt{(r+D / 2)^{2}-y^{2}}-(r+D / 2),
\end{aligned}
$$

where $r=\left[\left(c-\frac{D}{2}-\frac{t}{2}\right)^{2}-\left(\frac{D}{2}+\frac{t}{2}\right)^{2}\right] /(2 D)$.

- Part MK $(c / 2<y<c / 2+R)$ :

$$
\begin{aligned}
& x(y)=h-a \sqrt{1-\left(\frac{y-c / 2}{b}\right)^{2}}, \\
& z(y)=\sqrt{(r+D / 2)^{2}-y^{2}}-(r+D / 2),
\end{aligned}
$$

where $h=\left(\frac{c}{2}-R\right) \cdot \tan \left(\frac{\pi}{2}-\omega\right), R=\frac{c}{2}-\frac{t}{2}-\frac{D}{2}$, $\omega=\arctan \left(\frac{c-D \sin (\gamma)}{D}\right)$, and $\gamma=\arcsin \left(\frac{0.5 \cdot c}{r+0.5 \cdot D}\right)$.

- Part KA $(x(y=c / 2+R)<x<w / 4)$ :

$$
\begin{aligned}
& y(z)=\sqrt{(r+D / 2)^{2}-(z+r+D / 2)^{2}}, \\
& z(x)=O Z-\sqrt{A^{2}-(x-O X)^{2}},
\end{aligned}
$$

where $O X=w / 4, O Z=\frac{\left(x_{2}-O X\right)^{2}-\left(x_{1}-O X\right)^{2}+z_{2}^{2}-z_{1}^{2}}{2 \cdot\left(z_{2}-z_{1}\right)}$, and $A=\sqrt{\left(x_{1}-O X\right)^{2}+\left(z_{1}-O Z\right)^{2}}$. Additionally, $\left(x_{1}, z_{1}\right)$ and $\left(x_{2}, z_{2}\right)$ are the coordinates of two points in part MK with $y_{1}=c / 2+R-0.001$ and $y_{2}=$ $c / 2+R$.

In this work, eqs. (1) to (6) are implemented in Wolfram Mathematica, then the length of the loop is calculated symbolically as a function of the parameter $t$. Subsequently, the function FindMinimum is employed to find the value of $t$ that yields the minimum length of the yarn loop. For this article, we consider a plain weft knitted fabric studied in [18] that has the course space $c=0.4857$ $\mathrm{mm}$, the wale space $w=0.8327 \mathrm{~mm}$, and the yarn diameter $D=0.1845 \mathrm{~mm}$. The optimum value of $t$ is equal to $0.072 \mathrm{~mm}$, corresponding to the length of the loop of 2.742 mm (cf. fig. 3).
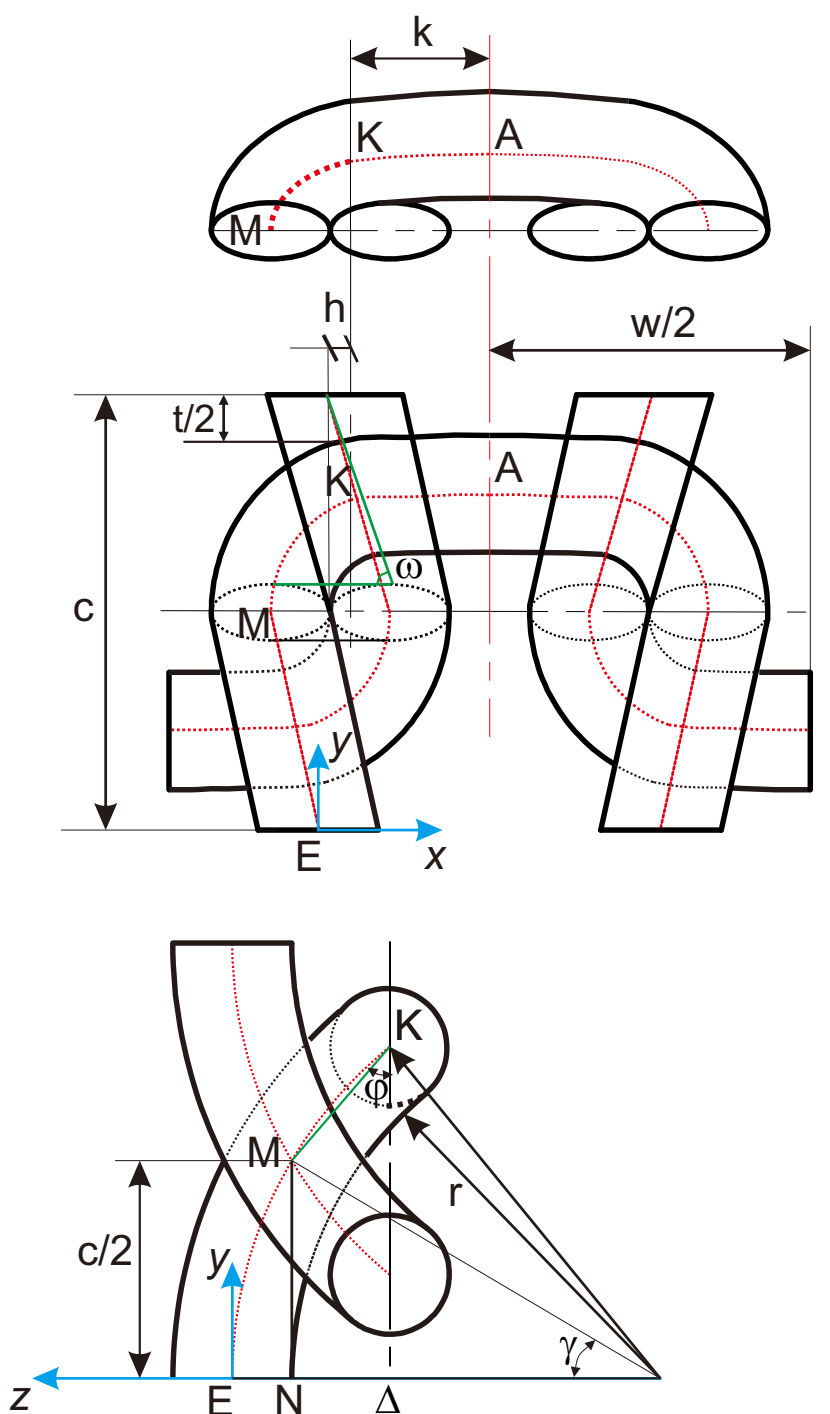

Figure 2: Different views of the idealized plain weft knitted fabric RUC proposed by Vassiliadis et al. 26] (from top to bottom: top view, front view and side view) 


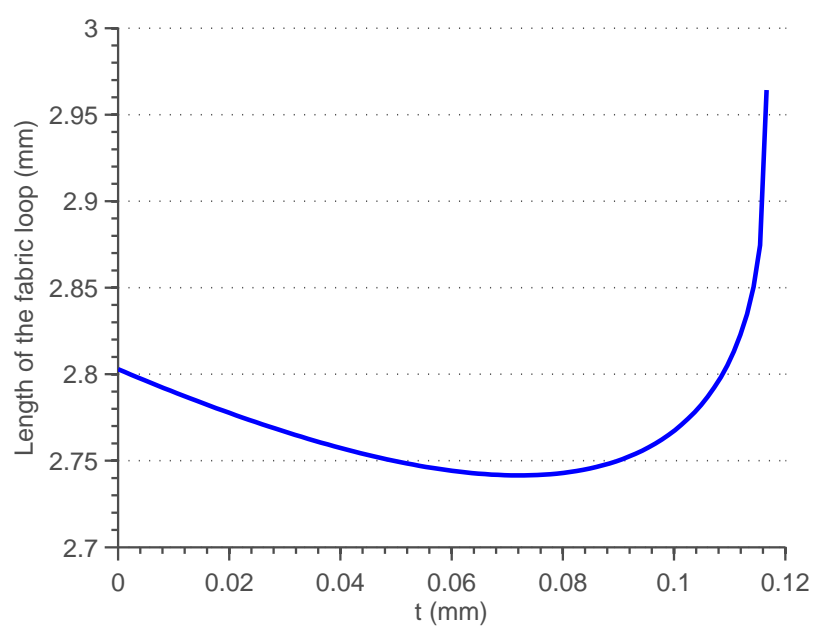

Figure 3: Relationship between the parameter $t$ and the fabric yarn loop.

\subsection{Material parameters}

For multifilament fabrics, the fabric yarn is considered as a transversely isotropic material, i.e., mechanical material behaviors are the same in the directions that are perpendicular to the fiber axis, but different along the fiber axis. To implement this model in ABAQUS, a local axis is defined along the heart line of the yarn as shown in fig. 4 Herein, the direction 3 is defined along the heart line of the yarn, while the direction 1 is the direction normal to the heart line and the direction 2 is defined so that it can comply with the other directions to create a right-handed, orthogonal coordinate system. The elastic parameters of the yarn utilized in this work are extracted from 18, and presented in table 1. It is worth mentioning here that in case of large deformation, hyperelasticity models, e.g., the hyperelasticity model proposed by Charmetant et al. [42, ,360 should be utilized for modeling the fabric yarn.

\section{Numerical homogenization method for knitted fabrics}

As mentioned in section 2, the macroscopic mechanical behavior of knitted fabrics is mainly governed by their constituents at meso and microscale. Therefore, it is quite cumbersome to phenomenologically model knitted fabrics. On the other hand, if we consider the fibers or yarns, i.e., the local heterogeneities, explicitly in the structural analysis, it will result in an extremely computationally demanding simulation. Multiscale modeling is an eligible solution for this case.

In general, multiscale methods can be divided into three categories, viz., hierarchical, semi-concurrent and concurrent methods 43. Among them hierarchical multiscale method has been successfully utilized to extract material properties of dry fabrics [39], coated fabrics [13] and textile composites [40]. The core of this multiscale method is the RUC-based homogenization technique. Using this component level) can be transferred to the fine scale (i.e., the mesoscale or the yarn level in this study) as boundary conditions. In return, the stress of the coarse scale can be obtained from the stress fields in the fine scale by using a certain homogenization scheme ( $c f$. fig. 5).

In the present work, we use periodic boundary conditions (PBCs) and the numerical homogenization method to exchange the information between the macro and meso scales. For the sake of completeness, here we recall the batechnique. Subsequently, their implementation in ABAQUS will be elaborately presented.

\subsection{Theoretical background of the hierarchical multiscale method}

Let $\left\{\mathbf{u}: \mathscr{B} \times \mathscr{R} \rightarrow \mathscr{R}^{3}\right\}$ denote the displacement field at a material point $\mathbf{x} \in \mathscr{B}$ of the mesostructure $\mathscr{B} \subset \mathscr{R}^{3}$. The (quasi-)static equilibrium equation at the mesoscale can be stated as follows:

$$
\frac{\partial \sigma_{i j}}{\partial x_{i}}=0
$$

Herein, we assume there is no body force in the system. In this study, the symmetric stress $\boldsymbol{\sigma}$ is related to the strain $\epsilon$ by the elastic constitutive relation

$$
\sigma_{i j}=C_{i j k l} \epsilon_{k l},
$$

where $C_{i j k l}$ is the fourth-order stiffness tensor and the strain $\epsilon$ is defined as

$$
\epsilon_{k l}=\frac{1}{2}\left(\frac{\partial u_{k}}{\partial x_{l}}+\frac{\partial u_{l}}{\partial x_{k}}\right)
$$

At the mesoscale of knitted fabrics, contact among the yarns are also involved. As a consequence, the following conditions are imposed [14].

- Non-penetration conditions in the normal direction:

$$
[u]_{n} \leq g, \quad \sigma_{n} \leq 0, \quad \sigma_{n}\left([u]_{n}-g\right)=0
$$

- Frictional stick and slip conditions in the tangential surface:

$$
\begin{aligned}
& \left|\boldsymbol{\sigma}_{t}\right|<G, \quad \Rightarrow \quad[\mathbf{u}]_{t}=0, \\
& \left|\boldsymbol{\sigma}_{t}\right|=G, \quad \Rightarrow \quad[\mathbf{u}]_{t}=-\lambda \boldsymbol{\sigma}_{t} .
\end{aligned}
$$

Herein, $u_{n}=\mathbf{u} \cdot \mathbf{n},[u]_{n}$ is the relative displacement in the direction of the outward normal to the contact surface, $g$ is the initial gap function between contacting surfaces, $\sigma_{n}=(\boldsymbol{\sigma} \cdot \mathbf{n}) \cdot \mathbf{n}, \boldsymbol{\sigma}_{t}=\boldsymbol{\sigma} \cdot \mathbf{n}-\sigma_{n} \mathbf{n}, G$ is the Tresca friction traction, $\mathbf{u}_{t}=\mathbf{u}-u_{n} \mathbf{n},[\mathbf{u}]_{t}$ is the relative displacements in the tangential direction to the contact surface, $\lambda$ is the frictional coefficient and $\mathbf{n}$ is the unit outward normal vector to $\partial \mathscr{B}$. In ABAQUS, to impose these contact 


\begin{tabular}{l|l|l|l}
\hline \hline$E_{1}=10 \mathrm{~N} / \mathrm{mm}^{2}$ & $G_{12}=3.85 \mathrm{~N} / \mathrm{mm}^{2}$ & $\nu_{12}=0.3$ & $\nu_{13}=0.0037$ \\
$E_{2}=10 \mathrm{~N} / \mathrm{mm}^{2}$ & $G_{23}=5 \mathrm{~N} / \mathrm{mm}^{2}$ & $\nu_{21}=0.3$ & $\nu_{31}=0.3$ \\
$E_{3}=800 \mathrm{~N} / \mathrm{mm}^{2}$ & $G_{31}=5 \mathrm{~N} / \mathrm{mm}^{2}$ & $\nu_{13}=0.0037$ & $\nu_{23}=0.0037$ \\
\hline \hline
\end{tabular}

Table 1: Elastic properties of the fabric yarn studied in this work.

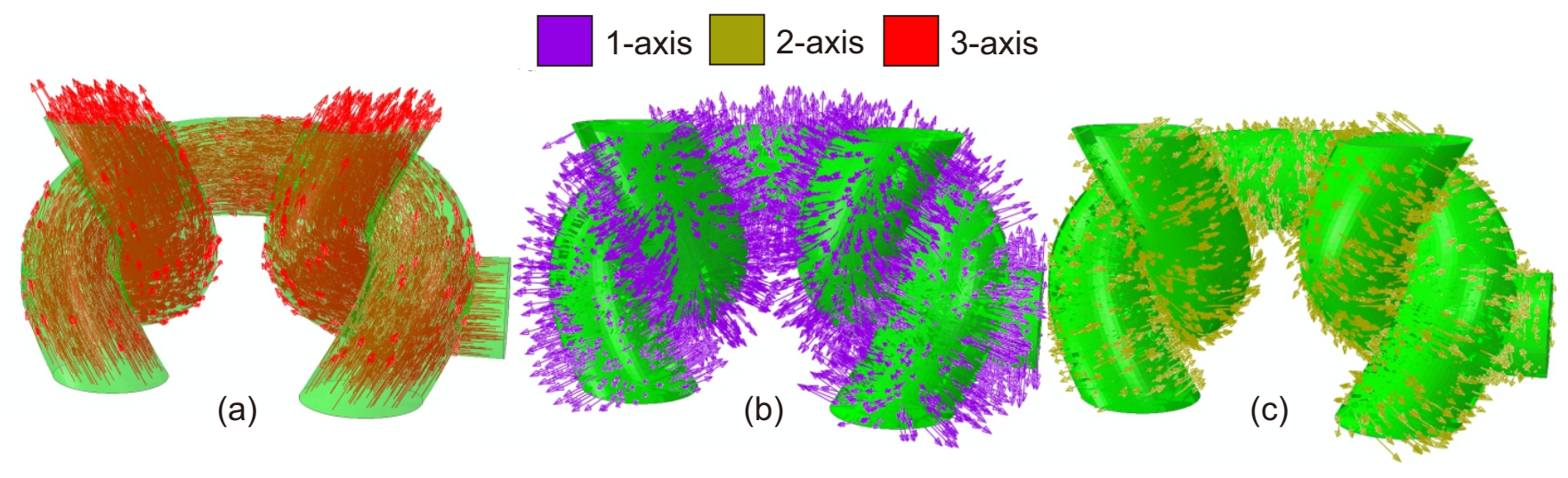

Figure 4: Material directions in the fabric yarn: (a) direction 3 is defined along the heart line, (b) direction 1 is defined perpendicularly to the heart line, and (c) direction 2 is defined in such a way that, together with other directions, it can form a right handed coordinate system.

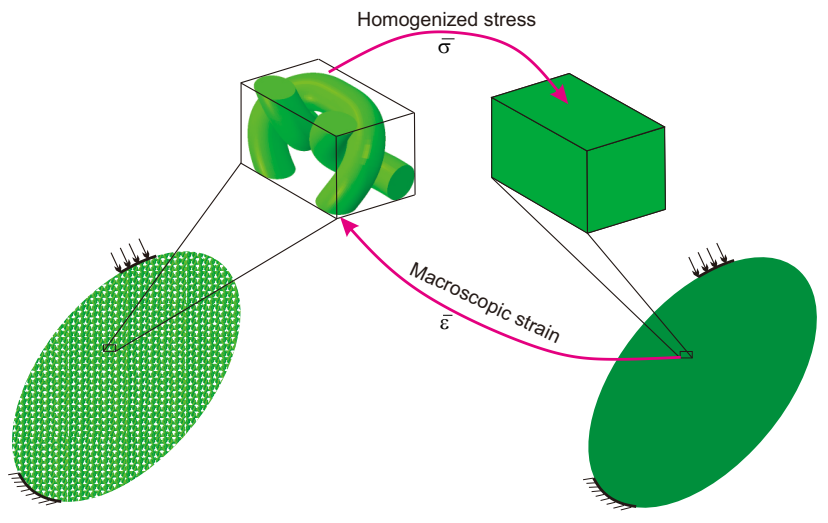

Figure 5: Homogenization method for knitted fabrics.

conditions we utilize the commands *Surface Behavior, pressure-overclosure=HARD and $*$ Friction [44.

These system of equations cannot be solved without ${ }_{390}$ certain boundary conditions. In RUC-based homogenization, these boundary conditions have to be formulated consistently with an averaging theorem [45. In this work, we use the averaging theorem formulated by Hill [46].

Let $\mathscr{V} \subset \mathscr{R}^{3}$ designate the RUC associated with the ${ }_{395}$ mesostructure $\mathscr{B}$. We define the overall macrostress $\overline{\boldsymbol{\sigma}}^{395}$ and the overall macrostrain $\overline{\boldsymbol{\epsilon}}$ of the mesostructure $\mathscr{B}$ as follows:

$$
\begin{aligned}
& \overline{\boldsymbol{\sigma}}=\frac{1}{|\mathscr{V}|} \int_{\mathbf{x} \in \mathscr{V}} \boldsymbol{\sigma}(\boldsymbol{x}) \mathrm{d} \mathscr{V} \\
& \overline{\boldsymbol{\epsilon}}=\frac{1}{|\mathscr{V}|} \int_{\mathbf{x} \in \mathscr{V}} \boldsymbol{\epsilon}(\boldsymbol{x}) \mathrm{d} \mathscr{V}
\end{aligned}
$$

where $|\mathscr{V}|$ is the volume of the RUC. The averaging theorem formulated by Hill requires that the average of the mesoscopic stress power to be equal to the macroscopic ${ }^{405}$ stress power. It leads to the following equation:

$$
\overline{\boldsymbol{\sigma}}: \dot{\overline{\boldsymbol{\epsilon}}}=\frac{1}{|\mathscr{V}|} \int_{\mathbf{x} \in \mathscr{V}} \boldsymbol{\sigma}(\boldsymbol{x}): \dot{\boldsymbol{\epsilon}}(\boldsymbol{x}) \mathrm{d} \mathscr{V}
$$

As a whole, there are three different boundary conditions for which eq. (14) is satisfied, viz., constant tractions, linear displacements and periodic displacements in conjunction with antiperiodic tractions, i.e., periodic boundary conditions [47]. These boundary conditions indeed manifest the macroscopic deformation modes with either prescribed overall macrostress $\overline{\boldsymbol{\sigma}}$ or prescribed overall macrostrain $\overline{\boldsymbol{\epsilon}}$. In this work, we employ the periodic boundary conditions in which we assume that the overall macrostrain $\overline{\boldsymbol{\epsilon}}$ is prescribed. Subsequently, the obtained stress fields in the deformed RUC will be utilized to calculate the overall macrostress $\overline{\boldsymbol{\sigma}}$ (cf. eq. 12 ).

\subsection{Periodic boundary conditions}

In this study, only the in-plane mechanical properties of the knitted fabric are considered. Thus, two-dimensional PBCs are imposed to the three-dimensional mesoscale model, i.e., the displacement in the thickness direction of the mesoscale model is left unassigned.

In practice, the PBCs are usually applied to the opposite surfaces of the RUC in a pointwise fashion. Specifically, in the case of the considered knitted fabrics ( $c f$. fig. 6), the PBCs are imposed on the material points in area $A_{1}$ and their counterparts in area $A_{3}$, which have the same values of $x_{1}$ and $x_{3}$. These conditions also apply for material points in area $A_{2}$ and their counterparts in area $A_{4}$, and those in area $A_{5}$ and area $A_{6}$.

We can express these PBCs for material points on each surface pair as follows: 


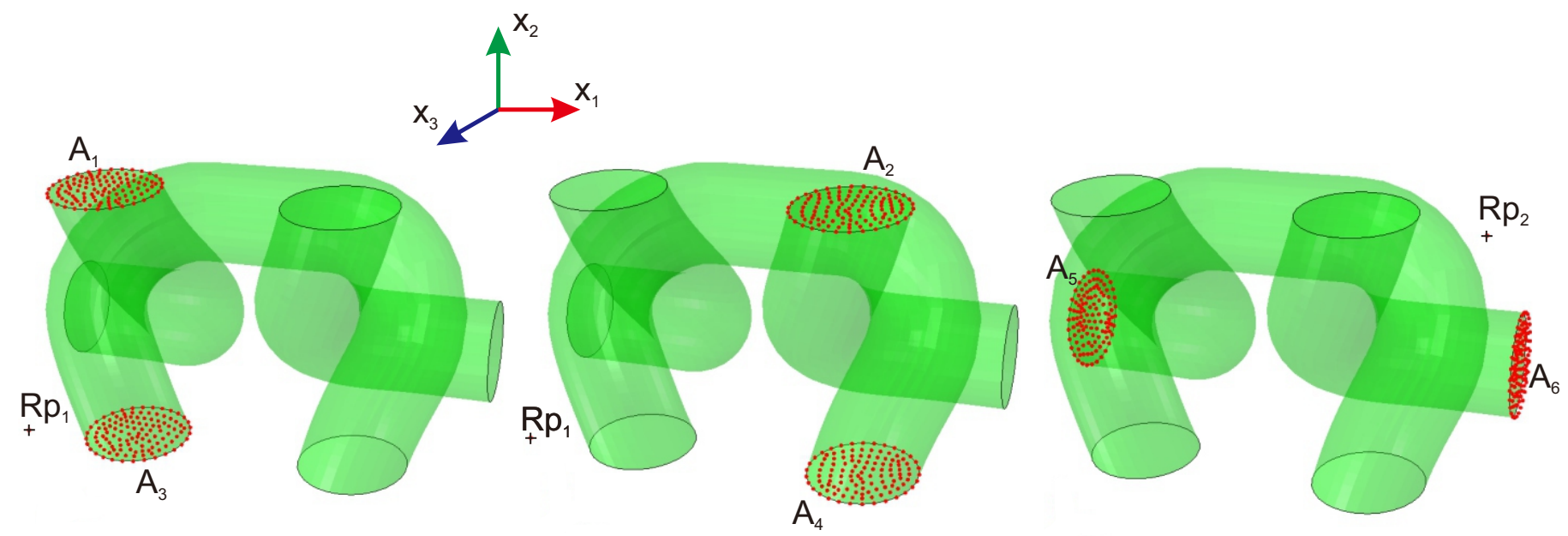

Figure 6: Areas in the knitted fabric model in which the $\mathrm{PBCs}$ are applied and locations of reference points $\left(\mathrm{Rp}_{1}\right.$ and $\mathrm{Rp}_{2}$ are respectively denoted for reference points 1 and 2), whose displacements control the deformation modes of the RUC.

- Surfaces $A_{1}$ and $A_{3}$ :

$$
\begin{aligned}
& u_{1}^{A_{1}}=u_{1}^{A_{3}}+\epsilon_{12} \cdot \Delta x_{2}, \\
& u_{2}^{A_{1}}=u_{2}^{A_{3}}+\epsilon_{22} \cdot \Delta x_{2}, \\
& u_{3}^{A_{1}}=u_{3}^{A_{3}}+\epsilon_{32} \cdot \Delta x_{2},
\end{aligned}
$$

- Surfaces $A_{2}$ and $A_{4}$ :

$$
\begin{aligned}
& u_{1}^{A_{2}}=u_{1}^{A_{4}}+\epsilon_{12} \cdot \Delta x_{2}, \\
& u_{2}^{A_{2}}=u_{2}^{A_{4}}+\epsilon_{22} \cdot \Delta x_{2}, \\
& u_{3}^{A_{2}}=u_{3}^{A_{4}}+\epsilon_{32} \cdot \Delta x_{2},
\end{aligned}
$$

- Surfaces $A_{6}$ and $A_{5}$ :

$$
\begin{aligned}
& u_{1}^{A_{6}}=u_{1}^{A_{5}}+\epsilon_{11} \cdot \Delta x_{1}, \\
& u_{2}^{A_{6}}=u_{2}^{A_{5}}+\epsilon_{21} \cdot \Delta x_{1}, \\
& u_{3}^{A_{6}}=u_{3}^{A_{5}}+\epsilon_{31} \cdot \Delta x_{1},
\end{aligned}
$$

where $u_{1}, u_{2}$ and $u_{3}$ are the displacements in $x_{1^{-}}, x_{2^{-}}$and $x_{3}$-directions, $\Delta x_{1}$ and $\Delta x_{2}$ are respectively the distances from surfaces $A_{1}$ to $A_{3}$ and $A_{6}$ to $A_{5}$ and $\epsilon_{i j}$ are the components of the macroscopic strain tensor. Equations (15) to 23) can be implemented in ABAQUS [44 by using the linear equation constraint. The generic form of the linear equation constraint in ABAQUS is as follows:

$$
A_{1} u_{i}^{P}+A_{2} u_{j}^{Q}+\cdots+A_{N} u_{k}^{R}=0,
$$

where $u_{i}^{P}$ is the nodal displacement at node $P$ in the $i$ direction, and $A_{\diamond}$ are coefficients that define the relative motion of the nodes [4]. To implement eqs. 15] to 23) 430 in ABAQUS using this linear equation constraint, we introduce two reference points ( $c f$. fig. 6), then the values of the last term in each equation are imposed as displacements on these reference points. It is noteworthy that these reference points are not connected to any material ${ }_{435}$ part, but deformation modes of the RUC are controlled by

\subsection{Numerical homogenization scheme}

The homogenized macroscopic stress tensor $\overline{\boldsymbol{\sigma}}$ can be calculated from eq. (12). This integration can be done numerically with respect to the utilized element type and element geometry. However, this approach is not numerically efficient. By using the divergence theorem and exploiting the equilibrium condition, eq. 12 can be expressed as:

$$
\bar{\sigma}_{\mathrm{ij}}=\frac{1}{|\mathscr{V}|} \int_{\mathbf{x} \in \partial \mathscr{V}} f_{\mathrm{j}} x_{\mathrm{i}} \mathrm{d} a,
$$

where $\mathbf{f}$ and $\mathbf{x}$ are respectively the traction and position vectors of material points on the surfaces of the RUC. In [48, it was proved that by using the PBCs eq. (23) can be simplified as follows:

$$
\bar{\sigma}_{\mathrm{ij}}=\frac{1}{|\mathscr{V}|} \int_{\mathbf{x} \in \partial \mathscr{V}} f_{\mathrm{j}} x_{\mathrm{i}} \mathrm{d} a=\frac{1}{|\mathscr{V}|} \sum_{p=1}^{3} f_{\mathrm{j}}^{\mathrm{p}} x_{\mathrm{i}}^{\mathrm{p}},
$$

where $\mathbf{f}^{\mathrm{p}}$ and $\mathbf{x}^{\mathrm{p}}$ are respectively the traction and position vectors of the reference points ( $c f$. fig. 6).

\section{Numerical results}

\subsection{Uniaxial tensile tests}

Physical uniaxial tensile tests in the wale and course directions of the same knitted fabric sample were performed in [18 using the Kawabata Evaluation System for Fabrics (KES-F). In these tests, the samples have rectangular shapes with a width of $2.5 \mathrm{~cm}$ and a length of $20 \mathrm{~cm}$ and were cut along the course and wale directions. These samples were then mounted between two rigid parallel bars. During the course of deformation, one bar was clamped, while the other was moved in parallel to the clamped one. The strain rate is constant and equal to $0.1 \mathrm{~mm} / \mathrm{s}$. This strain rate is quite slow, thus the inertial effect can be omitted in the analysis. The imposed deformation was stopped when the tensile load reached the value of 50 
$\mathrm{gf} / \mathrm{cm}$. It is noteworthy that during the course of tensile tests the change in thickness direction of fabrics is assumed to be negligible that is why the load per unit length is used instead of stresses.

\section{Tensile test along the course direction}

As mentioned in section 4.2 the deformation modes of the RUC are imposed through the displacements of the reference points. For the uniaxial test along the course direction, i.e., the coursewise uniaxial test, the boundary500 conditions are set as follows:

- The strain $\epsilon_{11}=0.15$ that results the displacement of $0.12 \mathrm{~mm}$ in the $x_{1}$-direction ( $c f$. fig. 6) is imposed at reference point 1 . Displacements in other directions ${ }_{505}$ at reference point 1 are fixed.

- At reference point 2 , displacement in the $x_{1}$ - and $x_{3}$-direction are fixed, while the displacement in the $x_{2}$-direction is free. Thus, it allows the lateral de-

The RUC is discretized by using three-dimensional brick elements, i.e., the C3D8 element in ABAQUS/Standard [44. A mesh sensitivity study is performed to ensure that ${ }_{520}$ a converged solution is obtained for successively refined meshes. Figure 7 illustrates five meshes used for this study containing $3,023,15,165,32,137,110,180$ and 249,893 elements. The corresponding load-strain curves obtained from these models, viz, Model 1 to Model 5, are plotted in ${ }_{525}$ fig. 8. Indeed, the load in these curves are calculated by the overall macrostress $\overline{\boldsymbol{\sigma}}$ to the thickness of the RUC. As can be seen from this graph, the load-strain curve shows no more visible change when the number of elements in the mesh is greater than 32,137 , i.e., Model $3_{53}$ ( $c f$. fig. 7f). Therefore, in subsequent simulations, Model

4753 will be utilized. Moreover, to efficiently exploit the parallel computing capacity of ABAQUS, simulations of Model 3 with different number of CPUs are performed. As can be seen from fig. 9, the simulation can be done within 569 seconds (wall clock time) when 8 CPUs (Intel Core i7$6900 \mathrm{~K}, 3.2 \mathrm{GHz}$ ) are utilized. The calculation time does not reduce when the number of utilized CPUs is further increased.

In ref. [18, the value of the frictional coeffient is missing, therefore we perform a parametric study to identify the value of frictional coefficient that can yield a good cor- ${ }_{540}$ relation between the numerical simulation and the experimental data from the coursewise tensile test. The loadstrain curves obtained from the parametric study when the frictional coefficient is changed from 0.05 to 0.2 are within the considered range of the frictional coefficient, the homogenized load-strain curves are in good correlation with the corresponding experimental data, but the homogenized load-strain curves in general are less nonlinear. At the beginning of the load step, the discrepancies among them are quite small. However, these discrepancies become more visible when the strain reaches $5 \%$. Among them, the difference between homogenized result obtained using $\mu=0.2$ and the experimental data at this strain level is the largest. When the strain passes $10 \%$, the discrepancies between experimental data and the homogenized results obtained using $\mu=0.15$ and $\mu=0.2$ are quite small. Therefore, considering the correlation between the homogenized result and the experimental data for the whole range of strain, the frictional coefficient of 0.15 is chosen. This value is also utilized for the subsequent simulations in this study.

The deformed configuration of the RUC at the end of the load step is plotted in fig. 11. As can be seen from fig. 11 , the maximum stress mainly occurs in part KM (cf. fig. 2) of the yarns. This part is quite straight, therefore, the material orientation, i.e., direction 3 in fig. 4 . is almost in line with the loading direction. Additionally, the material behavior in this direction is assumed linear, that is why the homogenized load-strain curves obtained in fig. 10 are quite linear compared to the corresponding experimental data. Moreover, it can be seen from fig. 11. that the strain in the transversal direction of the yarn, which results from the contact between the yarn head and yarn legs, is quite large compared to the strain in the fiber direction. Nevertheless, the corresponding stress is quite small ( $c f$. fig. 11d). It is indeed expected in this case because the Young's moduli in the transversal directions are quite small compared to the counterpart in the fiber direction ( $c f$. table 1). This result, however, emphasizes the importance of considering the anisotropic nature in mechanical behavior of the fabric yarn. Moreover, it is noteworthy that by leaving the degree of freedom in the $x_{2}$-direction at reference point 2 free, contraction in the lateral direction is allowed ( $c f$. fig. 11 ). Thus, the uniaxial stress state can be achieved in the RUC.

\section{Tensile test along the wale direction}

Similar to the tensile test in the course direction, the uniaxial stress state in the wale direction can be achieved by using the following boundary conditions:

- The strain $\epsilon_{22}=0.10$, which results in a displacement of $0.049 \mathrm{~mm}$ in the $x_{2}$-direction ( $c f$. fig. 6), is imposed at reference point 2. Displacements in other directions at reference point 2 are fixed.

- At reference point 1 , displacements in the $x_{2}$ - and $x_{3}$-directions are fixed, while the displacement in the $x_{1}$-direction is free. Thus, it allows the lateral deformation of the RUC during the course of loading. As a result, the RUC is subjected to uniaxial stress state. 

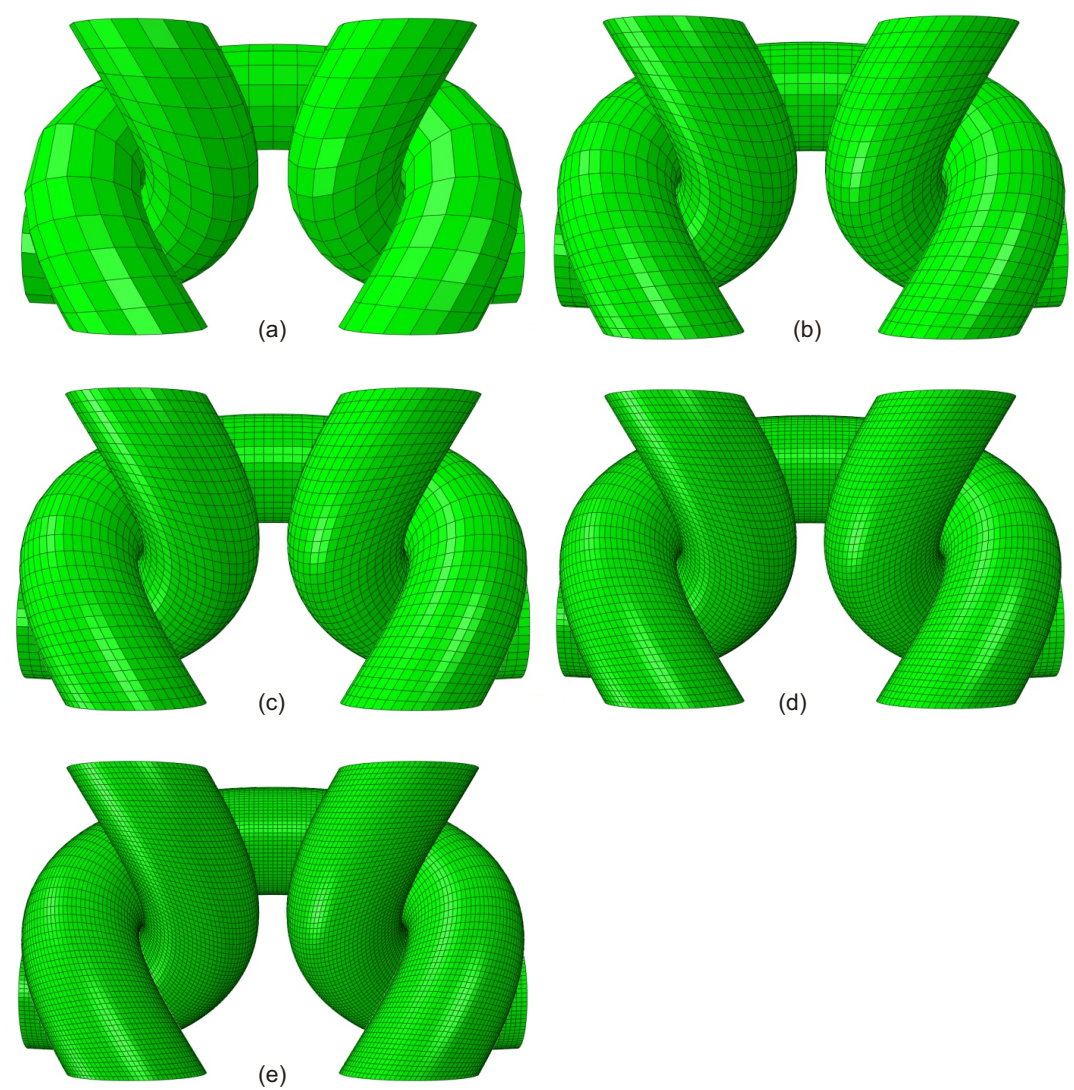

Figure 7: Different mesh densities are utilized to discretize the knitted fabric RUC: (a) Model 1: 3,023 elements, (b) Model 2: 15,165 elements, (c) Model 3: 32,137 elements, (d) Model 4: 110,180 elements, and Model 5: 249,893 elements.

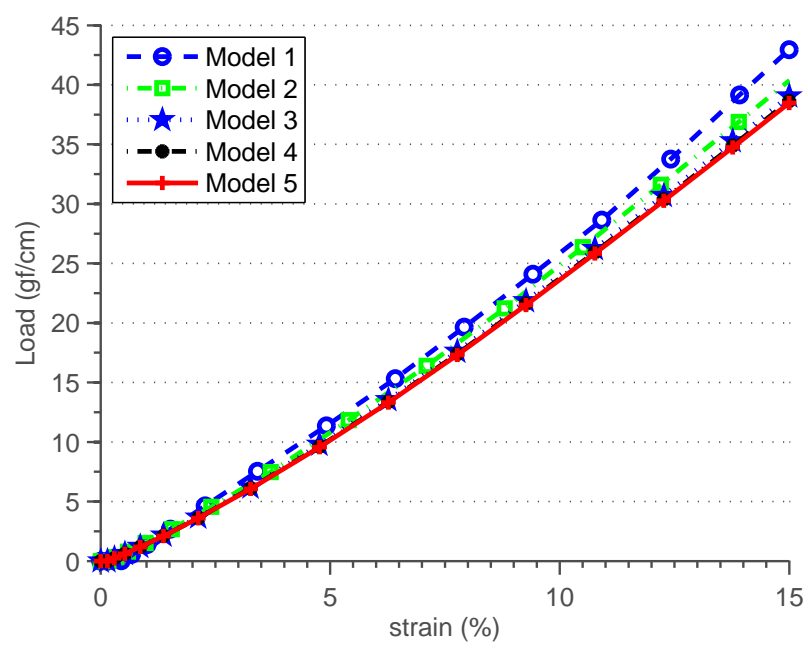

Figure 8: Homogenized load-strain curves obtained from the coursewise tensile test when different mesh densities ( $c f$. fig. 7) are utilized to discretize the RUC.

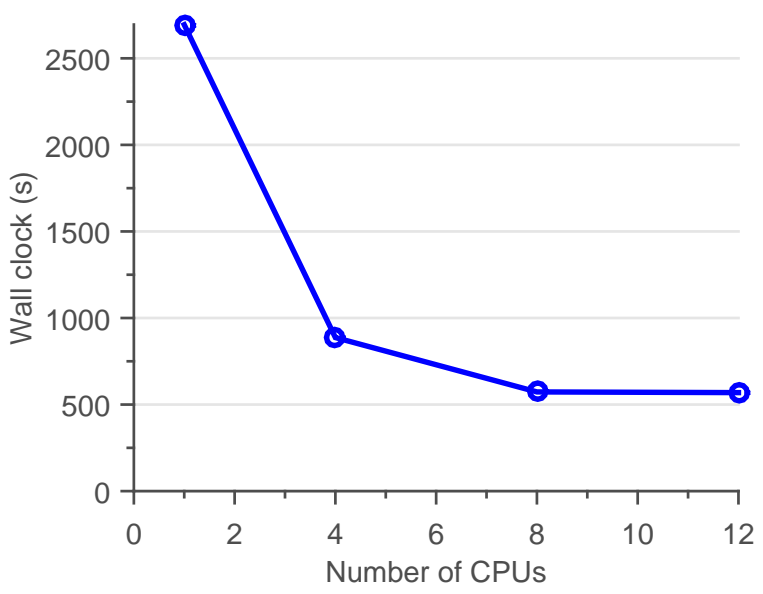

Figure 9: Calculation time vs. the number of CPUs that are utilized for Model 3 to simulate the uniaxial coursewise tensile test. 


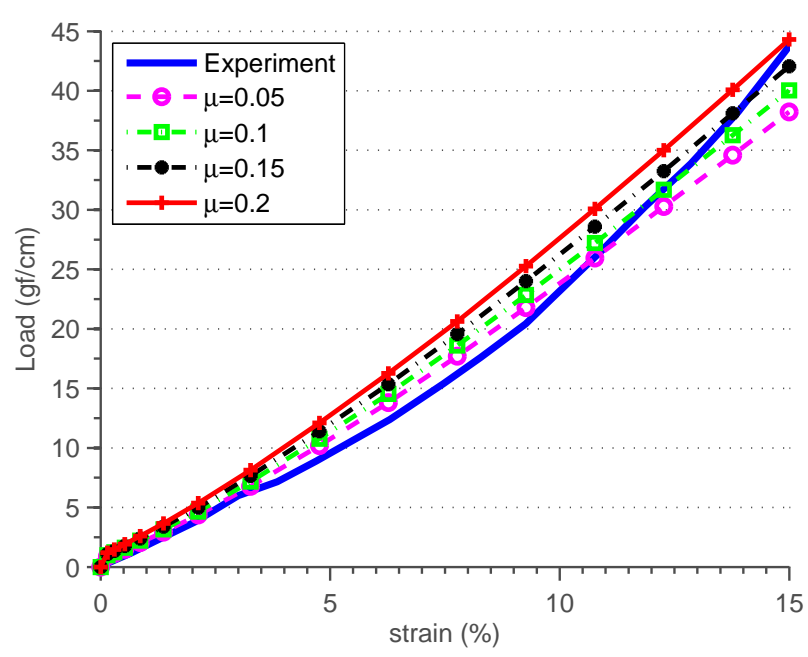

Figure 10: Effect of the frictional coefficient $\mu$ on the homogenized load-strain curves obtained from the coursewise tensile test.

- Moreover, to avoid rigid body motions, the displace- ${ }_{600}$ ments of a node located at the center of surface $A_{3}$ are fixed.

Additionally, it is worth repeating that during the course of simulation, Model 3 ( $c f$. fig. 7) is utilized with the frictional coefficient of 0.15 , as determined in the previous $_{605}$ section. The stress distribution on the deformed configuration of the RUC as well as its homogenized load-strain curve in this test can be seen in fig. 12 and fig. 13 , respectively. It is clear from fig. 13 that the model can capture well the nonlinear behavior of the knitted fabric. It thus ${ }_{610}$ proves the validity of the frictional coefficient chosen in the previous test. Compared to the previous test, this time the effect of the inter-yarn interaction is more pronounced. It manifests itself not only in a larger contact area in the fabric yarn ( $c f$. the non-zero contact-pressure bands in fig. 12 p and fig. 11d d), but also in the homogenized load-strain curve plotted in fig. 13 . In this graph, it can ${ }^{615}$ be seen that the homogenized load-strain curve is more nonlinear compared to the counterpart in fig. 10. This phenomenon originates from the contact friction among the yarn as mentioned in [14]. Moreover, it is noteworthy that the behavior of the fabric in the wale direction ${ }^{620}$ is stiffer than in the course direction. It is in fact in line with the observation presented in 2, where the authors mentioned that in plain weft knitted fabrics, the load can be transferred through wavy paths that are defined by the fabrics' loops and connection points ( $c f$. fig. 14). Indeed, the crimp level, i.e., the level of waviness, of the path in ${ }^{625}$ wale direction is less than the counterpart in the course direction. Eventually, it leads to the stiffer mechanical behavior of the knitted fabric in the wale direction.

\subsection{Shear test}

As mentioned in section 1, knitted fabrics can be used to their excellent formability. During the forming process, dry knitted fabrics can be subjected to a very complicated deformation, which is a combination of biaxial tension, inplane shear, transverse compaction and out-of-plane bending. Among them, the in-plane shear deformation is significant and can cause problems, such as the appearance of wrinkles [4]. Therefore, it is vital to understand the fabric shear behavior to model forming process with confidence.

In 18, a shear test of the same fabric studied in this 590 work has been done. The sample has dimensions of $20 \mathrm{~cm} \times$ $5 \mathrm{~cm}$. The sample was mounted between two rigid bars, as it was in the the tensile tests. Then one bar was clamped and the other was moved parallel to the clamped one. At first this bar was moved along the the wale direction to impose a pretension of $10 \mathrm{gf} / \mathrm{cm}$. Then, it was moved along the course direction to impart shear deformation on the sample. The shearing deformation is stopped when the shear angle is equal to $8^{\circ}$. For this test, the boundary conditions of the RUC are set as follows:

- At reference point 1 , the displacements in the $x_{1}$ and $x_{3}$-directions are fixed, while the displacement in the $x_{2}$-direction is free.

- At reference point 2, the displacement in the $x_{3}$ direction is fixed. A force of $0.00476 \mathrm{~N}$, which is equivalent to the pretension of $10 \mathrm{gf} / \mathrm{cm}$, is applied in the $x_{1}$-direction. Subsequently, the induced displacement in the $x_{1}$-direction was retained. Afterward, a strain $\epsilon_{12}$ of 0.14 , which results in a displacement of $0.116 \mathrm{~mm}$ in the $x_{2}$-direction, is imposed at reference point 2.

- Moreover, to avoid rigid body motion, displacements of a node located at the center of area $A_{5}$ are fixed.

The deformed configuration and the stress distribution on the RUC is visualized in fig. 15. It can be seen from this figure that the value of the von Mises stress in this test is quite small compared to the previous tests. In fig. 16 . the homogenized load-shear angle curve is plotted together with the corresponding experimental data. Generally, the prediction values are monotonically lower than the experimental data, but the discrepancy between them is quite small in this case. The reasons for this discrepancy can be as follows:

- The value of load in this test is ca. 10 times smaller than those in the walewise and coursewise uniaxial tests (the maximum value is $c a .0 .0588 \mathrm{~N}$ ). With this range of load, it can be rather challenging to measure the force precisely. This problem manifests itself in a quite noisy curve in fig. 16 .

- Moreover, the value of pretension force, which is very small ( $c a .0 .00476 \mathrm{~N}$ ) to measure precisely, can also affect the homogenized load-shear angle curve. 


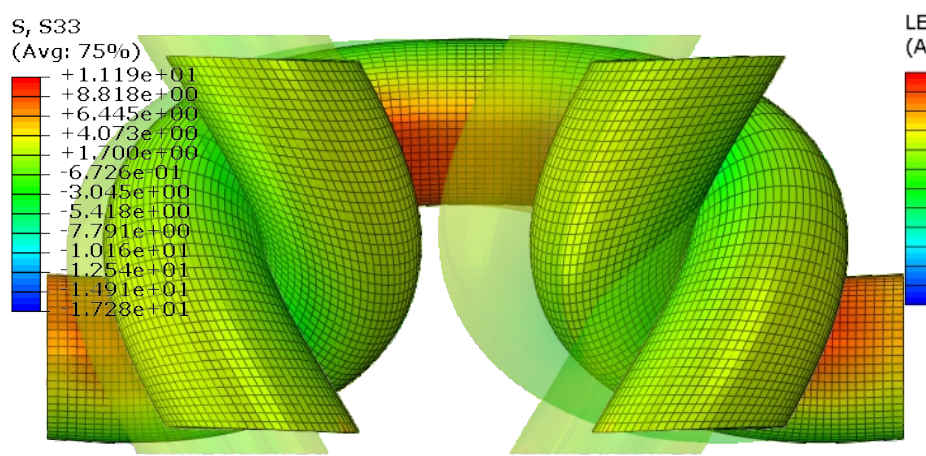

(a)

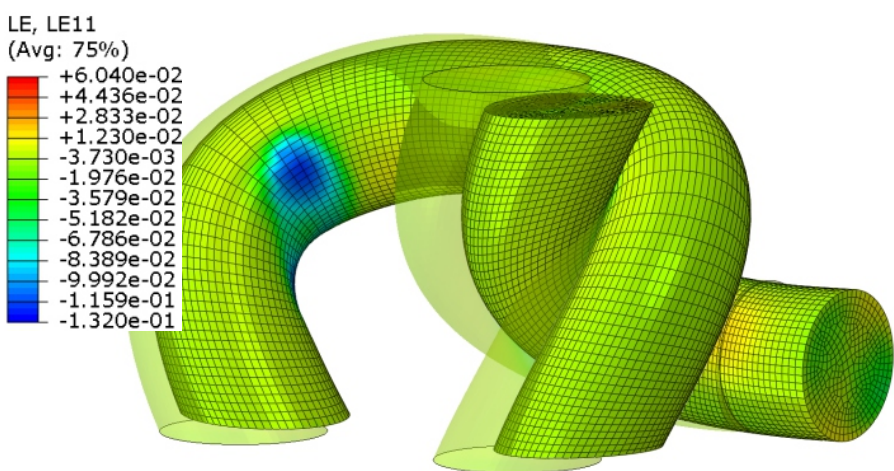

(c)

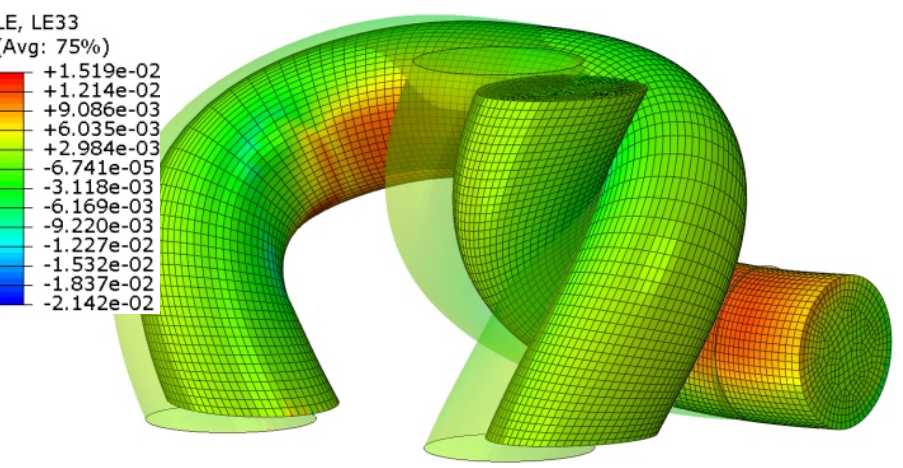

(b)

\section{CPRESS}

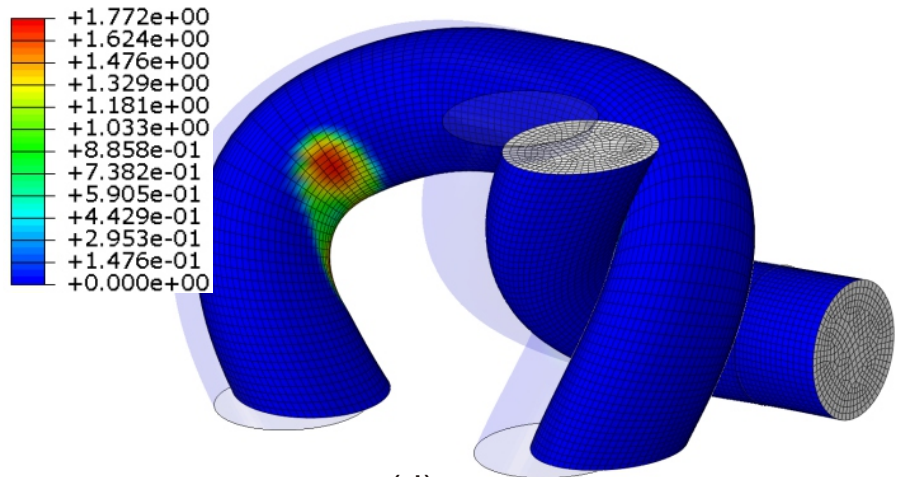

(d)

Figure 11: Stress and strains distribution on the fabric yarns: (a) stress in the fiber direction, (b) strain in the fiber direction, i.e., direction 3 in fig. 4 . (c) strain in direction 1, and (d) contact pressure between yarn head and yarn leg

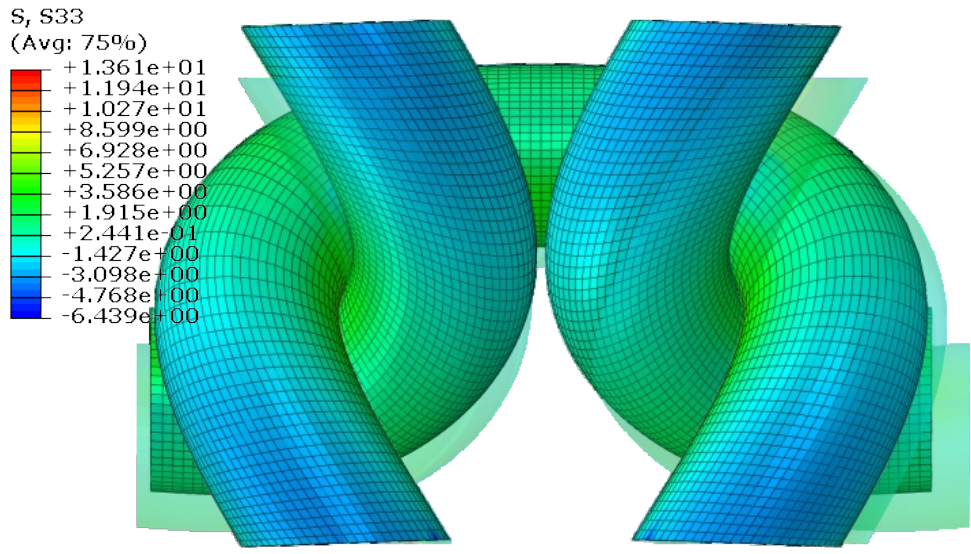

(a)

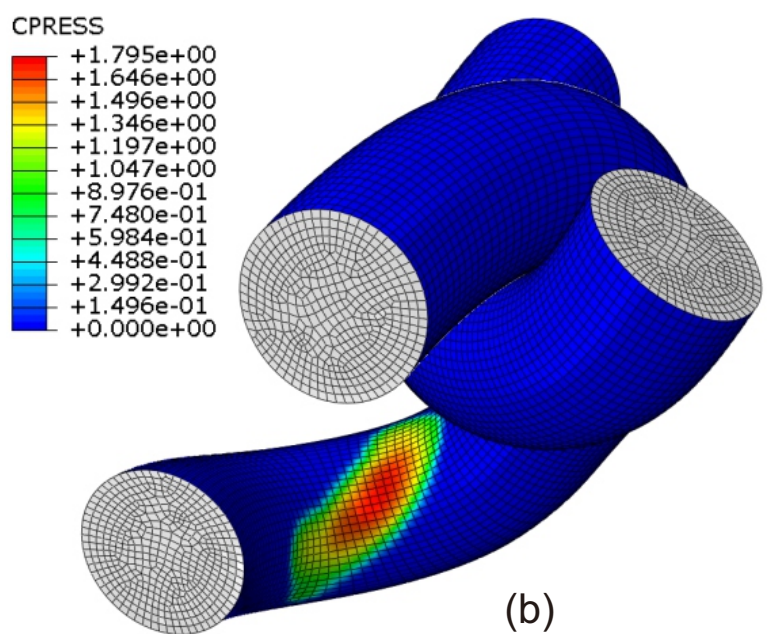

(b)

Figure 12: Stress distribution in the fiber direction on the fabric yarns and contact pressure, which results from the frictional contact between the fabric yarns in the walewise tensile test. 


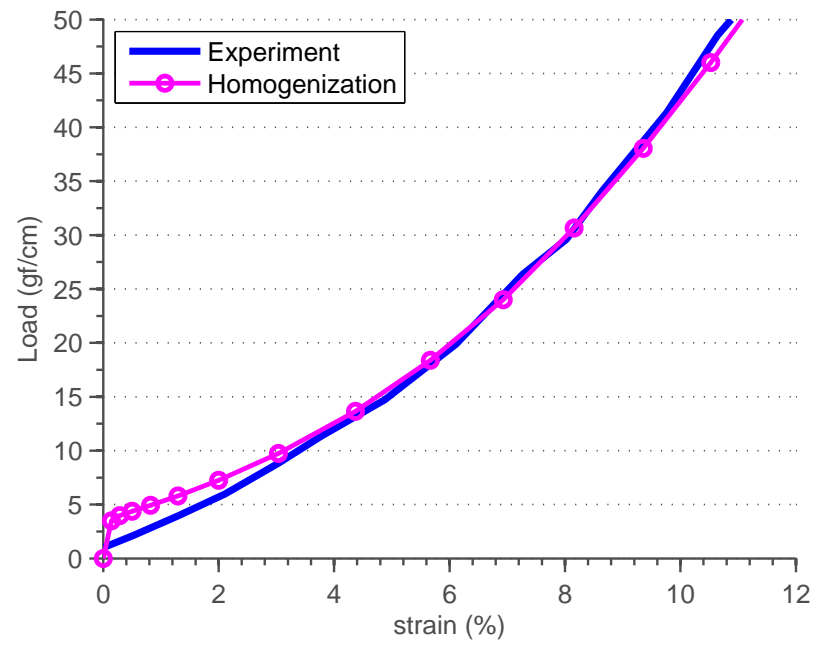

Figure 13: The homogenized load-strain curve obtained from the RUC and the corresponding experimental data in the walewise tensile test.

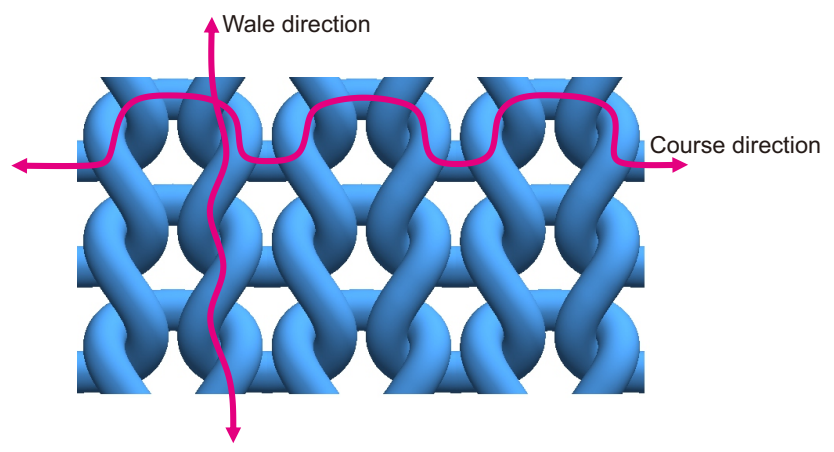

Figure 14: The load transfer paths in plain weft knitted fabrics defined in 2

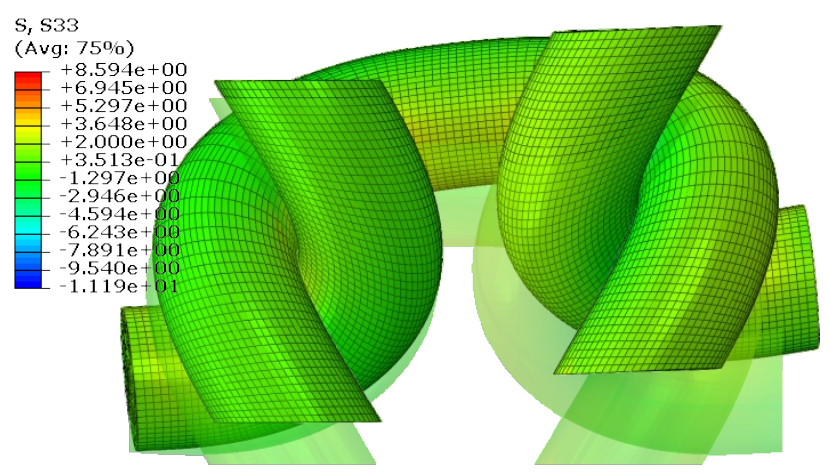

Figure 15: Stress distribution in the fiber direction on the fabric yarns in the shear test.

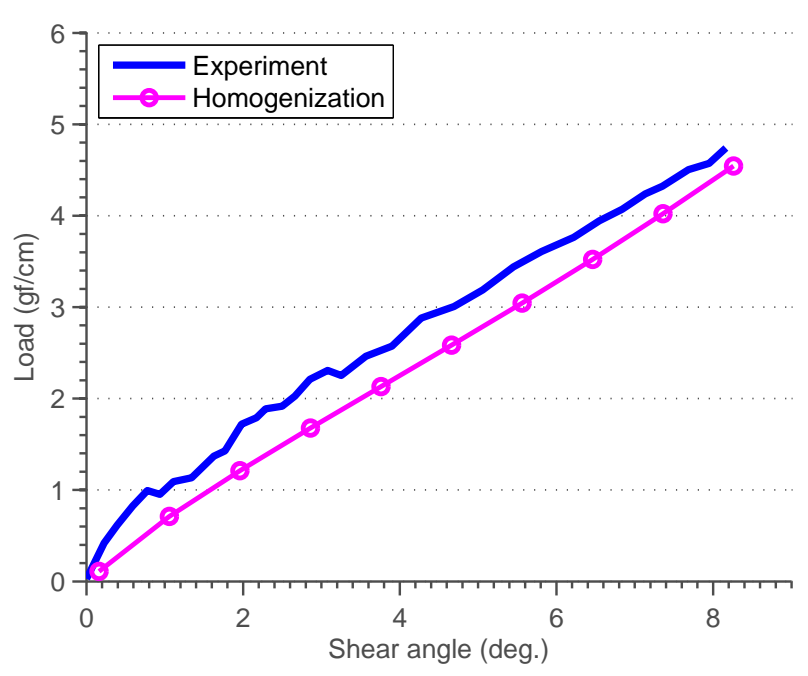

Figure 16: The homogenized load-shear angle and its corresponding experimental data in the shear test.

\subsection{Virtual biaxial tensile tests}

As a whole, the interaction between fabric yarns can affect significantly the mechanical behavior of knitted fabrics. Thus, biaxial tensile tests are essential to capture this effect. In literature, the behavior of fabrics is investigated on a cruciform sample [50]. During the course of the experiment, the strains at the center of the cruciform is recorded, while the stresses are derived from the forces applied at the arms of the cruciform. Indeed, these derived stresses are different from the stresses at the center of the cruciform. Thus, the obtained stress-strain curves do not reflect the constitutive behavior of the considered fabrics [13. As a consequence, it can lead to some difficulties to interpret these data and utilize them to develop a macroscopic material model for fabrics. Moreover, the number of biaxial tensile tests need to characterize the mechanical behavior of fabrics can be extensive and expensive. To overcome these difficulties in this section we utilize the proposed model to perform biaxial tensile tests for different course-wale strain ratios. The homogenized load-strain curves for different strain ratios, viz., 1:0, 2:1, $1: 2$ and $0: 1$, in the course and wale directions are shown in fig. 17. From the figure, it can be seen that the knitted fabrics behavior is highly anisotropic. These data can be useful for developing macroscopic constitutive models of knitted fabrics, which can be utilized for structural analyses.

\section{Conclusions}

660
The ultimate target of this article is to propose a numerical framework to predict the mechanical properties of knitted fabrics based on their mesostructure, the properties of their constituents, i.e., the fabric yarns, and the interaction among the fabric yarns. This has been achieved by using the hierarchical multiscale method. The core 


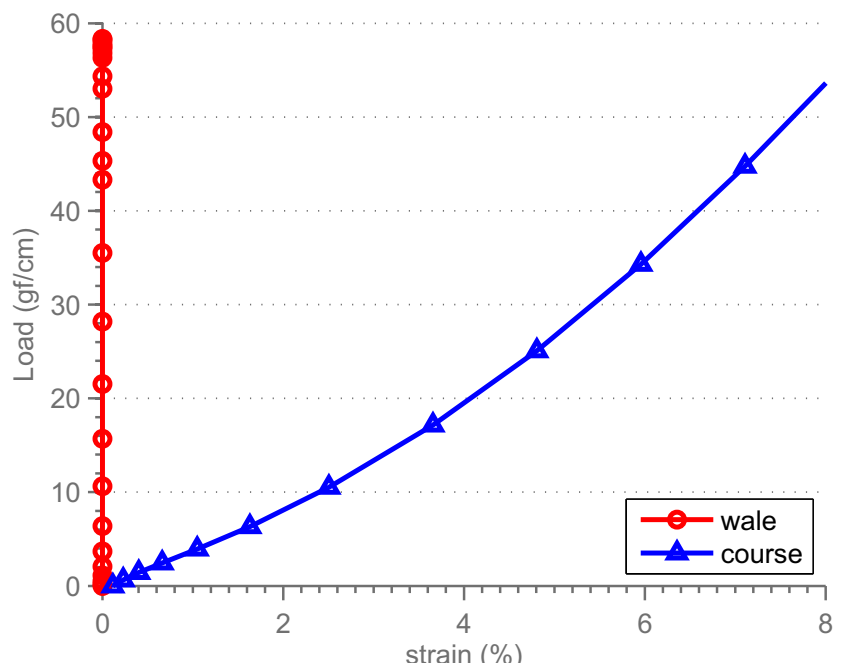

(a)

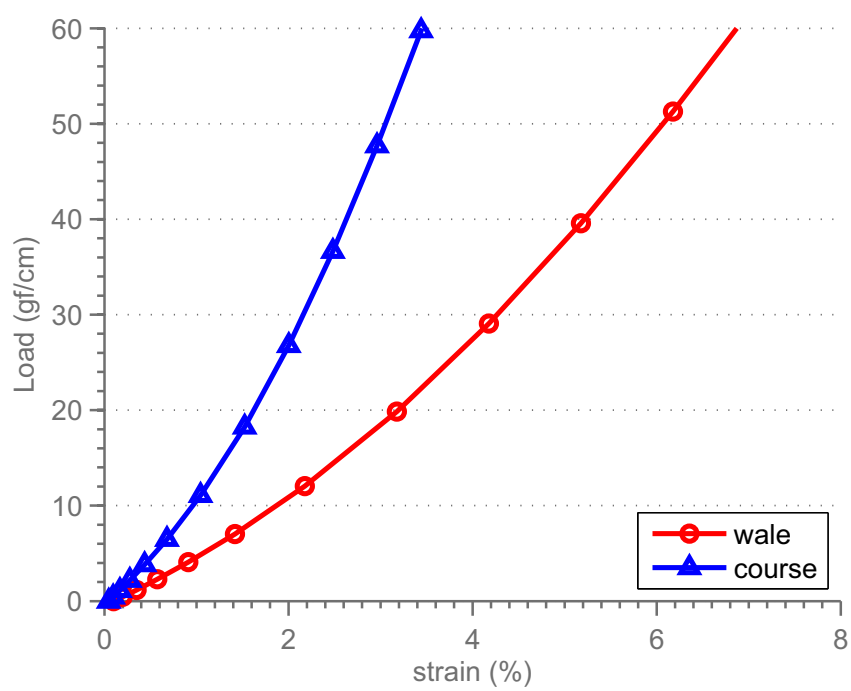

(c)

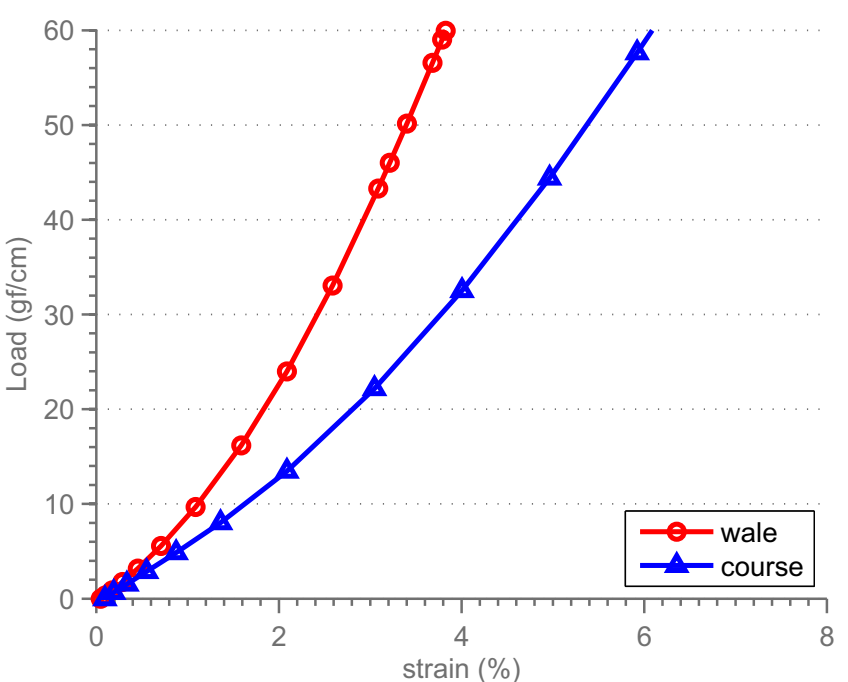

(b)

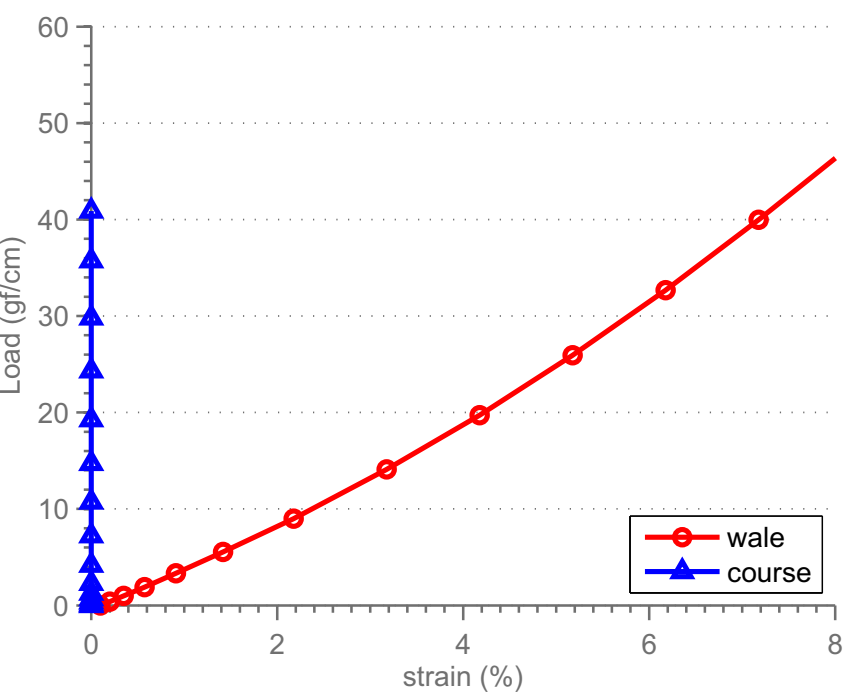

(d)

Figure 17: The homogenized load-strain curves in biaxial tests with different course-wale strain ratios: (a) Walewise strip biaxial test, i.e., $\epsilon_{11}: \epsilon_{22}=1: 0$, (b) $\epsilon_{11}: \epsilon_{22}=2: 1$, (c) $\epsilon_{11}: \epsilon_{22}=1: 2$, and $\epsilon_{11}: \epsilon_{22}=0: 1$, i.e., coursewise strip biaxial test. 
of our proposed framework is the nonlinear finite element analysis of the fabric RUC. As a starting point, the basic structural parameters of knitted fabrics, viz., the course space, the wale space, and the diameter of the fabric yarn, are utilized to create the geometry of the RUC. To cap- ${ }^{705}$ ture the fibrous nature of the fabric yarns, the transversed isotropic material model is employed to model the fabric yarns. During the course of the analysis, PBCs are imposed on the RUC, which allows the macroscopic defor- ${ }^{710}$ mation to be transferred to the RUC. Additionally, the frictional contact interaction among the fabric yarns is also considered. Afterward, the numerical homogenization scheme is utilized to extract the macroscopic stresses ${ }^{715}$ from the stresses in the deformed RUC. In several numerical studies considering uniaxial tension, shear, and biaxial tension, it has been shown that the predicted mechanical behavior of knitted fabrics are in good agreement with ${ }^{720}$ the corresponding experimental data. Furthermore, the nonlinear finite element simulations could be done in a reasonable time. Further research will apply this numerical framework for different knitted fabrics that have more ${ }^{725}$ sophisticated mesostructures to predict their mechanical properties in small or moderate strain regimes, as we have done in this work, and later expand it to finite deformation regime. Moreover, employing the second order homog- ${ }^{730}$ enization method to simulate the nonlinear out-of-plane deformation mode is also an intriguing extension.

\section{Acknowledgment}

The authors acknowledge funding through the SUTD Digital Manufacturing and Design (DManD) Centre, sup- ${ }_{740}$ ported by the Singapore National Research Foundation. The first author, Tien-Dung Dinh, would like to express his sincere acknowledgment to Prof. dr. ir. Wim Van Paepegem, Ghent University, Belgium for his excellent ${ }_{745}$

\section{References}

[1] T. D. Dinh, A. Rezaei, S. Puystiens, M. Van Craenenbroeck, K. Carbonez, L. De Laet, M. Mollaert, D. Van Hemelrijck, W. Van Paepegem, A study of tension fabric membrane structures under in-plane loading: Nonlinear finite element analysis and validation Composite Structures 128 (2015) 10-20. doi: $10.1016 / \mathrm{j}$.compstruct.2015.03.055

11 URL http://www.sciencedirect.com/science/article/pii/ S0263822315002287

[2] D. Liu, D. Christe, B. Shakibajahromi, C. Knittel, N. Castaneda, D. Breen, G. Dion, A. Kontsos, On the role of

- material architecture in the mechanical behavior of knitted textiles International Journal of Solids and Structuresdoi:10.1016/j.ijsolstr.2017.01.011

URL //www.sciencedirect.com/science/article/pii/ S0020768317300112

[3] M. Araujo, R. Fangueiro, H. Hong, Modelling and simulation of the mechanical behaviour of weft-knitted fabrics for technical applications Autex Research Journal 4 (2). URL http://faculty.mu.edu.sa/public/uploads/ 1430144753.6268\%D8\%A7\%D8\%B3\%D8\%AA\%D8\%AE\%D8\%AF\%D8\%A7\%D9\% 85\%D8\%A7\%D8\%AA\%20\%D8\%B5\%D9\%86\%D8\%A7\%D8\%B9\%D9\%8A\%D8\%A9. pdf

[4] H. Wang, J. F. O'Brien, R. Ramamoorthi, Data-driven elastic models for cloth: modeling and measurement ACM Press, 2011, p. 1. doi:10.1145/1964921.1964966

11 URL http://portal.acm.org/citation.cfm?doid=1964921. 1964966

${ }_{n}$ [5] J. Abel, J. Luntz, D. Brei, Hierarchical architecture of active knits Smart Materials and Structures 22 (12) (2013) 125001. doi:10.1088/0964-1726/22/12/125001. URL http://stacks.iop.org/0964-1726/22/i=12/a=125001

[6] S. V. Lomov, M. Moesen, R. Stalmans, G. Trzcinski, J. V. Hum-

beeck, I. Verpoest, Finite element modelling of SMA textiles: superelastic behaviour The Journal of The Textile Institute 102 (3) (2011) 232-247. doi:10.1080/00405001003696464 URL http://dx.doi .org/10.1080/00405001003696464

[7] M. S. Yeoman, D. Reddy, H. C. Bowles, D. Bezuidenhout, P. Zilla, T. Franz, A constitutive model for the

warp-weft coupled non-linear behavior ofknittedbiomed\begin{tabular}{ll|lllll}
\hline ical textiles & Biomaterials & 31 & $(32)$ & $(2010)$ & $8484-8493$.
\end{tabular} doi:10.1016/j.biomaterials.2010.07.033

1] URL //www.sciencedirect.com/science/article/pii/ S0142961210008744

[ [8] K. H. Leong, S. Ramakrishna, Z. M. Huang, G. A. Bibo, The potential of knitting for engineering compositesa review, Composites Part A: Applied Science and Manufacturing 31 (3) (2000) 197-220. doi:10.1016/S1359-835X (99)00067-6

URL http://www.sciencedirect.com/science/article/pii/ S1359835X99000676

[9] M. Glazzard, P. Breedon, Weft-knitted auxetic textile design physica status solidi (b) 251 (2) (2014) 267-272. doi:10.1002/pssb.201384240

URL http://onlinelibrary.wiley.com/doi/10.1002/pssb. 201384240/abstract

[10] M. J. Abghary, H. Hasani, R. J. Nedoushan, Numerical simulating the tensile behavior of 11 rib knitted fabrics using a novel geometrical model Fibers and Polymers 17 (5) (2016) 795-800. doi:10.1007/s12221-016-5791-6

760 URL

http://link.springer.com/10.1007/

[11] Z.-M. Huang, S. Ramakrishna, Modeling mechanical proper-

1. ties of knitted fabric composites-Part I: Overview and Geometric Description Science \& Engineering of Composite Materials 10 (3) (2002) 163-188.

IIUL https://www. degruyter.com/downloadpdf/j/secm. 2002. $10.3 / \mathrm{secm} .2002 .10 .3 .163 / \mathrm{secm} .2002 .10 .3 .163 . \mathrm{xml}$

[12] A. U. Loginov, S. A. Grishanov, R. J. Harwood, Modelling the LoadExtension Behaviour of Plain-knitted Fabric: Part I:

770 A Unit-cell Approach towards Knitted-fabric Mechanics. The

n. Journal of The Textile Institute 93 (3) (2002) 218-238. doi: 
$10.1080 / 00405000208630566$

URL http://dx.doi .org/10.1080/00405000208630566

[13] T. D. Dinh, A. Rezaei, L. Daelemans, M. Mollaert, 845 D. Van Hemelrijck, W. Van Paepegem, A Hybrid Micromeso-scale Unit Cell Model for Homogenization of the Nonlinear Orthotropic Material Behavior of Coated Fabrics Used in Tensioned Membrane Structures Composite Structures doi: 10.1016/j.compstruct.2016.12.027

780 URL http://www.sciencedirect.com/science/article/pii/ S0263822316313204

[14] S. Fillep, J. Orlik, Z. Bare, P. Steinmann, Homogenization in periodically heterogeneous elastic bodies with multiple micro-contact, Mathematics and Mechanics of Solids 19 (8) 855 (2014) 1011-1021. doi:10.1177/1081286513501104.

URL http://journals.sagepub.com/doi/abs/10.1177/ 1081286513501104

[15] P. J. McKee, A. C. Sokolow, J. H. Yu, L. L. Long, E. D. Wetzel, Finite element simulation of ballistic impact on sin-360 790 gle jersey knit fabric Composite Structures 162 (2017) 98-107. doi:10.1016/j.compstruct.2016.11.086

URL http://www.sciencedirect.com/science/article/pii/ S0263822316308340

[16] S. Seyedin, M. S. Romano, A. I. Minett, J. M. Razal, Towards 365 the Knittability of Graphene Oxide Fibres, Scientific Reports 5 (2015) 14946. doi:10.1038/srep14946

URL http://www.nature.com/srep/2015/151013/srep14946/ full/srep14946.html

[17] B. Hagège, P. Boisse, J.-L. Billoët, Finite element analyses 370 of knitted composite reinforcement at large strain Revue Européenne des Eléments Finis 14 (6-7) (2005) 767-776. doi: 10.3166/reef .14.767-776

URL https://www.tandfonline.com/doi/full/10.3166/reef . 14.767-776

[18] S. G. Vassiliadis, A. E. Kallivretaki, C. G. Provatidis, Mechanical simulation of the plain weft knitted fabrics, International Journal of Clothing Science and Technology 19 (2) (2007) 109-130. doi:10.1108/09556220710725711

URL http://www.emeraldinsight.com/doi/full/10.1108/38 09556220710725711

[19] H. Hong, M. D. De Araujo, R. Fangueiro, O. Ciobanu, Theoretical analysis of load-extension properties of plain weft knits made from high performance yarns for composite reinforcement $\mid$ Textile research journal 72 (11) (2002) 991-996.885 URL http://journals.sagepub.com/doi/abs/10.1177/ 004051750207201110

[20] E. Gravas, P. Kiekens, L. Langenhove, Predicting fabric weight per unit area of single-and double-knitted structures using appropriate software Autex Res. J 6 (4) (2006) 223-237.

URL http://autexrj.com/cms/zalaczone_pliki/5-06-4.pdf

[21] E. Tompkins, The science of knitting, Wiley, 1914

22] J. Chamberlain, Hosiery Yarns and Fabrics, Vol. II, (City of Leicester College of Technology, UK), 1926.

[23] F. Peirce, Geometrical Principles Applicable to the Design of 995 Functional Fabrics, Textile Research Journal 17 (3) (1947) 123147. doi:10.1177/004051754701700301 URL http://dx.doi.org/10.1177/004051754701700301

[24] G. A. V. Leaf, A. Glaskin, 43The Geometry of a Plain Knitted Loop Journal of the Textile Institute Transactions 46 (9) (1955)900 T587-T605. doi:10.1080/19447027.1955.10750345 URL http://dx.doi.org/10.1080/19447027.1955.10750345

[25] S. Ramakrishna, Characterization and modeling of the tensile properties of plain weft-knit fabric-reinforced composites, Composites Science and Technology 57 (1) (1997) 1-22. doi:y05 10.1016/S0266-3538(96)00098-X

URL http://www.sciencedirect.com/science/article/pii/ S026635389600098X

[26] S. G. Vassiliadis, A. E. Kallivretaki, C. Provatidis, Geometrical modelling of plain weft knitted fabrics 32 (2007) 62-71. 910 URL http://nopr.niscair.res.in/bitstream/123456789/ 406/1/FTR $\% 2032 \% 281 \% 29 \% 20 \% 282007 \% 29 \% 2062-71$. pdf

[27] M. J. Abghary, R. J. Nedoushan, H. Hasani, Multi-Scale
Modeling the Mechanical Properties of Biaxial Weft Knitted Fabrics for Composite Applications Applied Composite Materials (2016) 1-16doi:10.1007/s10443-016-9551-y

URL s10443-016-9551-y

http://link.springer.com/article/10.1007/

[28] N. Shekarchizadeh, M. M. Abedi, R. Jafari Nedoushan, Prediction of elastic behavior of plain weft-knitted composites Journal of Reinforced Plastics and Composites 35 (22) (2016) 1613-1622. doi:10.1177/0731684416661639

URL http://journals.sagepub.com/doi/abs/10.1177/ 0731684416661639

[29] M. Duhovic, D. Bhattacharyya, Simulating the deformation mechanisms of knitted fabric composites Composites Part A: Applied Science and Manufacturing 37 (11) (2006) 1897-1915. doi:10.1016/j.compositesa.2005.12.029

URL http://www.sciencedirect.com/science/article/pii/ S1359835X06000133

[30] P. Popper, The theoretical behavior of a knitted fabric subjected to biaxial stresses, Textile Research Journal 36 (2) (1966) 148-157.

URL http://journals.sagepub.com/doi/pdf/10.1177/ 004051756603600208

[31] W. J. Shanahan, R. Postle, 23a Theoretical Analysis of the Tensile Properties of Plain-Knitted Fabrics. Part I: The LoadExtension Curve for Fabric Extension Parallel to the Courses, The Journal of The Textile Institute 65 (4) (1974) 200-212. doi:10.1080/00405007408630448

URL http://dx.doi .org/10.1080/00405007408630448

[32] G. Dusserre, L. Balea, G. Bernhart, Elastic properties prediction of a knitted composite with inlaid yarns subjected to stretching: A coupled semi-analytical model Composites Part A: Applied Science and Manufacturing 64 (2014) 185-193. doi:10.1016/j.compositesa.2014.05.007

10 URL http://www.sciencedirect.com/science/article/pii/ S1359835X14001420

[33] G. Dusserre, Modelling the hysteretic wale-wise stretching behaviour of technical plain knits, European Journal of Mechanics - A/Solids 51 (2015) 160-171. doi:10.1016/j.euromechsol. 2014.12.009

URL http://www.sciencedirect.com/science/article/pii/ S0997753814001909

[34] J. Abel, J. Luntz, D. Brei, A two-dimensional analytical model and experimental validation of garter stitch knitted shape memory alloy actuator architecture Smart Materials and Structures 21 (8) (2012) 085011. doi:10.1088/0964-1726/21/8/085011. URL http: //stacks . iop.org/0964-1726/21/i=8/a=085011

[35] F. Ji, R. Li, Y. Qiu, Simulate the Dynamic Draping Behavior of Woven and Knitted Fabrics Journal of Industrial Textiles 35 (3) (2006) 201-215. doi:10.1177/1528083706055753 URL http://journals.sagepub.com/doi/abs/10.1177/ 1528083706055753

[36] C. J. Chuong, Y. C. Fung, Three-dimensional stress distribution in arteries, Journal of Biomechanical Engineering 105 (3) (1983) $268-274$

[37] X. Peng, J. Cao, A dual homogenization and finite element approach for material characterization of textile composites Composites Part B: Engineering 33 (1) (2002) 45-56. doi: 10.1016/S1359-8368(01)00052-X

URL http://www.sciencedirect.com/science/article/pii/ S135983680100052X

[38] N. Takano, Y. Ohnishi, M. Zako, K. Nishiyabu, Microstructurebased deep-drawing simulation of knitted fabric reinforced thermoplastics by homogenization theory, International Journal of Solids and Structures 38 (3637) (2001) 6333-6356. doi: 10.1016/S0020-7683(00)00418-2

II URL https://www.sciencedirect.com/science/article/pii/ S0020768300004182

[39] V. Carvelli, C. Poggi, A homogenization procedure for the numerical analysis of woven fabric composites Composites Part A: Applied Science and Manufacturing 32 (10) (2001) 1425-1432. doi : $10.1016 / \mathrm{S} 1359-835 \mathrm{X}(01) 00041-0$ 

S1359835X01000410

[40] S. Jacques, I. De Baere, W. Van Paepegem, Application of periodic boundary conditions on multiple part finite element meshes for the meso-scale homogenization of textile fabric composites Composites Science and Technology 92 (2014) 41-54. doi:10.1016/j.compscitech.2013.11.023

920

URL http://www.sciencedirect.com/science/article/pii/ S0266353813004594

[41] E. Syerko, S. Comas-Cardona, C. Binetruy, Models of me-

1. chanical properties/behavior of dry fibrous materials at various

925 scales in bending and tension: A review, Composites Part A: Applied Science and Manufacturing 43 (8) (2012) 1365-1388. doi:10.1016/j.compositesa.2012.03.012

II URL http://www.sciencedirect.com/science/article/pii/ S1359835X12001054

930 [42] A. Charmetant, E. Vidal-Sallé, P. Boisse, Hyperelastic modelling for mesoscopic analyses of composite reinforcements, Composites Science and Technology 71 (14) (2011) 1623-1631. doi:10.1016/j.compscitech.2011.07.004 S0266353811002351

[43] H. Talebi, M. Silani, S. P. A. Bordas, P. Kerfriden, T. Rabczuk, A computational library for multiscale modeling of material failure Computational Mechanics 53 (5) (2014) 1047-1071. doi:10.1007/s00466-013-0948-2

940 URL http://link.springer.com/10.1007/ s00466-013-0948-2

[44] Hibbit, Karlsson, Sorensen, ABAQUS/Standard Analysis User's Manual, Hibbit, Karlsson, Sorensen Inc., 2014.

[45] C. Miehe, A. Koch, Computational micro-to-macro transitions of discretized microstructures undergoing small strains Archive of Applied Mechanics 72 (4-5) (2002) 300-317. doi:10.1007/s00419-002-0212-2.

URL http://link.springer.com/article/10.1007/ s00419-002-0212-2

950 [46] R. Hill, F. R. S, On constitutive macro-variables for heterogeneous solids at finite strain Proc. R. Soc. Lond. A 326 (1565) (1972) 131-147. doi:10.1098/rspa.1972.0001

URL http://rspa.royalsocietypublishing.org/content/ 326/1565/131

955 [47] M. Kästner, G. Haasemann, J. Brummund, V. Ulbricht, Com-

1. putation of effective stiffness properties for textile-reinforced composites using X-FEM, in: Mechanical Response of Composites, Springer, 2008, pp. 261-279.

URL http://link.springer.com/chapter/10.1007/ 978-1-4020-8584-0_13

[48] M. G. D. Geers, V. G. Kouznetsova, W. a. M. Brekelmans, Computational homogenization, in: R. Pippan, P. Gumbsch (Eds.), Multiscale Modelling of Plasticity and Fracture by Means of Dislocation Mechanics, no. 522 in CISM International Centre for Mechanical Sciences, Springer Vienna, 2010, pp. 327-394, dOI: 10.1007/978-3-7091-0283-1_7.

URL http://link.springer. com/chapter/10.1007/ 978-3-7091-0283-1_7

[49] J. Cao, R. Akkerman, P. Boisse, J. Chen, H. Cheng, E. de Graaf, J. Gorczyca, P. Harrison, G. Hivet, J. Launay, W. Lee, L. Liu, S. Lomov, A. Long, E. de Luycker, F. Morestin, J. Padvoiskis, X. Peng, J. Sherwood, T. Stoilova, X. Tao, I. Verpoest, A. Willems, J. Wiggers, T. Yu, B. Zhu,

- Characterization of mechanical behavior of woven fabrics:

975 Experimental methods and benchmark results Composites Part A: Applied Science and Manufacturing 39 (6) (2008) 1037-1053. doi:10.1016/j.compositesa.2008.02.016

URL http://linkinghub.elsevier.com/retrieve/pii/ S1359835X08000572

980 [50] T. D. Dinh, A. Rezaei, L. De Laet, M. Mollaert, D. Van Hemelrijck, W. Van Paepegem, A new elasto-plastic material model for coated fabric Engineering Structures 71 (2014) 222-233. doi:10.1016/j.engstruct.2014.04.027

URL http://www.sciencedirect.com/science/article/pii/ 